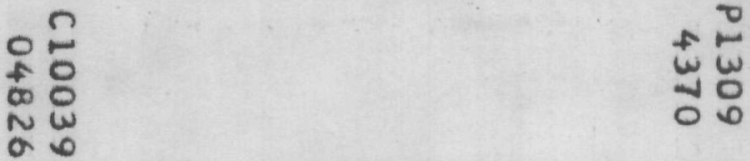




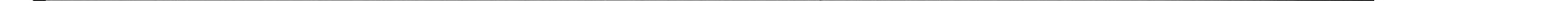


APPLICATION OF GASKINETICS TO SOME FLOW PROBLEMS 



\title{
APPLICATION OF GASKINETICS TO SOME FLOW PROBLEMS
}

\author{
PROEFSCHRIFT
}

TER VERKRIJGING VAN DE GRAAD VAN DOCTOR IN DE TECHNISCHE WETENSCHAPPEN AAN DE TECHNISCHE HOGESCHOOL DELFT, OP GEZAG VAN DE RECTOR MAGNIFICUS DR. IR. C. J. D. M. VERHAGEN, HOOGLERAAR IN DE AFDELING DER TECHNISCHE NATUURKUNDE, VOOR EEN COMMISSIE UIT DE SENAAT TE VERDEDIGEN OP WOENSDAG 9 OKTOBER 1968 TE 14 UUR

DOOR

\section{SOHAN LAL SARIN}

$$
\text { geboren te Jullunder, India }
$$

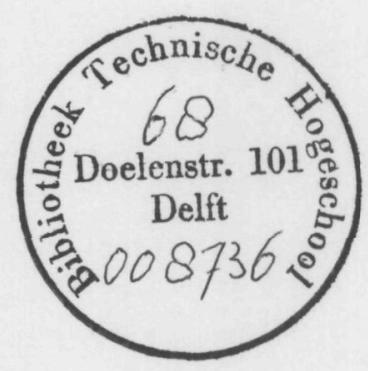

UITGEVERIJ WALTMAN - DELFT 
DIT PROEFSCHRIFT IS GOEDGEKEURD DOOR DE PROMOTOR PROF. DR. R. TIMMAN. 
To my Mother 



\section{CONTENTS}

Chapter 1 PROLOGUE. . . . . . . . . . . . . . . . 9

\section{Chapter 2 GENERAL INTRODUCTION}

2.1 The Boltzmann integro-differential equation . . . . . . . 11

2.2 Enumeration of the methods of solution of the Boltzmann equation ............... . . 12

2.2.1 The method of successive approximations . . . . . . 12

2.2.2 The method of models . . . . . . . . . . 15

2.2.3 The method of moments . . . . . . . . . 16

2.2.4 The variational method using a restricted variational principle . . . . . . . . . . . . . . 20

2.2.5 Numerical procedures . . . . . . . . . . 20

2.3 Conclusion . . . . . . . . . . . . . . . . . . . 21

\section{Chapter 3 COLLISION OF GAS CLOUDS}

3.1 Introduction . . . . . . . . . . . . 22

3.2 Formulation of the problem . . . . . . . . . . 22

3.3 Conservation equations and consistency relations . . . . . . 23

3.4 Transport equation . . . . . . . . . . . . . 25

3.5 Nondimensionalization . . . . . . . . . . . . . . 28

3.6 Solution and discussion . . . . . . . . . . . . . . . . . . . . . . . 29

3.7 Concluding remarks . . . . . . . . . . . . . . 30

Chapter 4 MODIFIED TRANSFER EQUATION APPROACH AND ITS APPLICATION TO LINEARIZED PLANE COUETTE FLOW

Part I Modified transfer equation approach

4.1 Introduction . . . . . . . . . . . . . . 31

4.2 Preliminary remarks prior to the introduction of the present method .............. . 31

4.3 Basic theory of the present method . . . . . . . . . 32

Part II Modified transfer equation method for linearized plane couette flow

4.4 Formulation of the problem . . . . . . . . . . 33

4.5 Choice of the distribution function . . . . . . . . . 34 
4.6 Calculation of the coefficients $\alpha_{i}^{\mp}(\mathrm{y})$. . . . . . . . . 35

4.6.1 Maxwell's equation of transfer . . . . . . . . . 35

4.6.2 Calculation of $\alpha_{1}^{\mp}(\mathrm{y}) \ldots \ldots \ldots 77$

4.6.3 Calculation of $\alpha_{2}^{\mp}$ (y) . . . . . . . . . . . . . . . 40

Chapter 5 KINETIC THEORY OF SHOCK STRUCTURE

5.1 Introduction . . . . . . . . . . . . . . 42

5.2 Basic equation and relations . . . . . . . . . . 43

5.3 General method of solution ... . . . . . . . . 46

Appendices. . . . . . . . . . . . . . . 51

References ................ . . 55

Summary ................. . . . 57

Samenvatting . . . . . . . . . . . . . . 59 


\section{Chapter 1}

\section{PROLOGUE}

The first germs regarding the growth of the kinetic theory of gases were discerned in the works of Hooke and Daniel Bernoulli. This all happened in the first half of the eighteenth century. There is usually a lull before a storm. The saying is probably applicable to theories as well. Nearly a century later after Bernoulli spurt of workers appeared on the scene and out of them signal contributions to the theory were made by Maxwell and Boltzmann. In fact, Maxwell and Boltzmann brought to bear upon the whole subject mathematical methods "far in advance of anything previously attempted on the subject". Maxwell discovered and, in part established theoretically the law of the distribution of velocities among the molecules of a gas (Maxwell's law). Further "Maxwell's work gave the first accurate theoretical determinations of the coefficients of viscosity, thermal conduction and diffusion in gases, for any molecular model". Boltzmann, on the other hand, derived the integro-differential equation which the velocity distribution must satisfy, whatever, the state of the gas or the forces acting on it. The solution of this equation for the state of equilibrium in a uniform gas is just the Maxwell function. This provided a rigorous derivation for the Maxwell velocity distribution.

After Maxwell and Boltzmann had put the first stones in the edifice of the kinetic theory of gases, a hunt began for techniques which could solve the Boltzmann equation; whole expeditions were on the go, with intricate gear and equipment. Results (though approximate) came in avalanches and these are surveyed in chapter 2 of this thesis.

The present intense interest in the kinetic theory of gases stems mainly from the possibility of flight at extreme altitudes and speeds. The kinetic theory is essentially superseded by the methods of statistical mechanics when the gas is in equilibrium. However, when a gas is not in equilibrium, an extension of techniques of kinetic theory provides methods for dealing with such a phenomenon as conduction in gases. The aim of the kinetic theory of gases is to predict the dynamics of a gas on the basis of molecular mechanics. To this end, the kinetic theory applies the laws of probability theory to the mechanical problems. Attention is directed on the state of the entire mass of gas: its temperature, density, pressure and other characteristics. There is no need to determine the velocities of the separate molecules. All characteristics of the state of gas should refer to the whole system of molecules as an assembly.

Before lowering the curtain on the present chapter it seems imperative to give in a nutshell the plan of the thesis.

In addition to the present chapter the thesis consists of four more chapters. A gen- 
eral survey of the existing methods of solution of the Boltzmann equation is presented in chapter 2. Some new techniques are developed in chapters 3-5 and with the aid of these certain specific problems in rarefied gas flows are investigated. Moreover, throughout the present work a monatomic gas is considered. The peculiarity of such a gas is that the molecule is identical with the atom. 


\section{GENERAL INTRODUCTION}

\subsection{The Boltzmann integro-differential equation}

The Boltzmann equation forms the starting point for many important investigations in the area of rarefied gas flows. The Boltzmann equation is an equation for the distribution function $f(\mathbf{r}, \mathbf{c}, t)$ of a gas. Here $f(\mathbf{r}, \mathbf{c}, t) d \mathbf{c} d \mathbf{r}$ is the probable (average) number of molecules which, at time $t$, are situated in the volume element $\mathbf{r}, d \mathbf{r}$ and have velocities lying in the range $\mathbf{c}, d \mathbf{c}$. The equation is an integro-differential equation for the distribution function $f(\mathbf{r}, \mathbf{c}, t)$ of a gas and reads in the absence of external forces:

$$
\frac{\partial f}{\partial t}+\mathbf{c} \cdot \frac{\partial f}{\partial \mathbf{r}}=\iiint\left(f^{\prime} f_{1}^{\prime}-f f_{1}\right) \mathrm{g} b d b d \varepsilon d \mathbf{c}_{1},
$$

where $b$ is the impact parameter, $\varepsilon$ is the azimuth of the plane of the relative orbit about an axis along the line of head on approach and $g$ is the magnitude of the relative velocity $\mathbf{g}$ of a colliding pair. Further quantities associated with second of a pair of molecules are denoted by subscript one; quantities associated with the outcome of an encounter, by primes; and the accents when attached to function symbols refer to their arguments,

$$
f^{\prime}=f\left(\mathbf{r}, \mathbf{c}^{\prime}, t\right) ; \quad f_{1}^{\prime}=f\left(\mathbf{r}, \mathbf{c}_{1}^{\prime}, t\right) .
$$

In $(2,1)$ the first term in the integrand is known as the emission term or inverse collision integral and represents the rate of gain or emission of molecules into a chosen velocity element while the second term in $(2,1)$ represents the rate of efflux or absorption of molecules from the chosen velocity element and is known as the absorption term or the direct collision integral. In calculating the change due to collisions in $(2,1)$ only binary encounters are considered. Further in considering the encounters between molecules having velocities within assigned ranges, it is assumed that both sets of molecules are distributed at random, and without any correlation between velocity and position, in the neighbourhood of the point $\mathbf{r}$. Also the interval $d t$ is supposed short compared with the scale of time-variation of macroscopic properties, but large compared with the duration of an encounter.

The equation $(2,1)$ is sometimes written in the symbolic form as

$$
\mathscr{D} f=J\left(f f_{1}\right)
$$


where $\mathscr{D}$ stands for the operator $\partial / \partial t+\mathbf{c} \cdot \partial / \partial \mathbf{r}$ while $J\left(f f_{1}\right)$ denotes the collision integral $\iiint\left(f^{\prime} f_{1}^{\prime}-f f_{1}\right) \mathrm{g} b d b d \varepsilon d \mathbf{c}_{1}$. The derivation of $(2,1)$ may be found in [1,2 and 3].

Observable properties of the gas are obtained from suitable averages over the distribution, which can be calculated when the distribution $f(\mathbf{r}, \mathbf{c}, t)$ is known. Thus the problem mainly boils down to the determination of the solution of the Boltzmann equation $(2,1)$.

The Boltzmann equation is extremely intricate and seldom susceptible to direct mathematical solution. The solution of the Boltzmann equation is, in general, a matter of considerable difficulty even in cases corresponding to the physically simplest situations. There exist numerous approximate methods of solution of equation $(2,1)$ and discussion of these methods forms the main theme of the subsequent sections of the present chapter. The difficulties inherent in the womb of the Boltzmann equation are also exposed.

\subsection{Enumeration of the methods of solution of the Boltzmann equation}

While dealing with the Boltzmann equation, difficulties encountered mainly depend upon two reasons. Firstly, it is due to the non-linearity of the Boltzmann equation. Secondly, the arguments of the distribution functions $f^{\prime}$ and $f_{1}{ }^{\prime}$ contain $\mathbf{c}$ and $\mathbf{c}_{1}$ in an inextricable way so that unless the explicit form of the distribution function $f$ is known, no integration can be effected.

One of the first systematic procedures to solve the Boltzmann equation was developed by Lorentz in connection with his theory of electrical conductivity of metals. Lorentz examined the case of a mixture of very massive and very small particles with so few of the smaller species that the number of their collisions with each other was small compared with the numbers with the larger particles. In this case the equation for the distribution function becomes a linear integro-differential equation.

Broadly speaking, the various methods hitherto employed in studying the boundary value or the initial value problems connected with the Boltzmann equation $(2,1)$ can roughly be enumerated as

a. The method of successive approximations.

b. The method of models.

c. The method of moments.

d. The variational method using a restricted variational principle.

e. Numerical procedures.

We will sketch the salient features of each of these methods in the following subsections.

\subsubsection{The method of successive approximations}

The methods of Enskog-Chapman and Hilbert belong to this category. In these 
methods we look for the Hilbert class of solutions (the so-called normal solutions) of the Boltzmann equation $(2,1)$ where the time dependence of the distribution function $f(\mathbf{r}, \mathbf{c}, t)$ is only via the five macroscopic quantities:

$$
f(\mathbf{r}, \mathbf{c}, t) \rightarrow f(\mathbf{r}, \mathbf{c} \mid n(\mathbf{r}, t), \mathbf{u}(\mathbf{r}, t), T(\mathbf{r}, t))
$$

where the vertical bar indicates that $f$ is a functional of $n(\mathbf{r}, t), \mathbf{u}(\mathbf{r}, t)$ and $T(\mathbf{r}, t)$ as far as its time dependence is concerned. The quantities $n(\mathbf{r}, t), \mathbf{u}(\mathbf{r}, t)$ and $T(\mathbf{r}, t)$ are defined as follows:

$$
\begin{aligned}
& n(\mathbf{r}, t)=\int f(\mathbf{r}, \mathbf{c}, t) d \mathbf{c} \\
& \mathbf{u}(\mathbf{r}, t)=\frac{1}{n(\mathbf{r}, t)} \int f(\mathbf{r}, \mathbf{c}, t) \mathbf{c} d \mathbf{c} \\
& \frac{3}{2} k T(\mathbf{r}, t)=\frac{1}{n(\mathbf{r}, t)} \int f(\mathbf{r}, \mathbf{c}, t) \frac{1}{2} m(\mathbf{c}-\mathbf{u})^{2} d \mathbf{c}
\end{aligned}
$$

In Enskog's method of solution [1] a solution of the form

$$
f=\frac{1}{\theta} f^{(0)}+f^{(1)}+\theta f^{(2)}+\ldots
$$

is assumed of the Boltzmann equation $(2,2)$ which is written as

$$
\xi(f)=J\left(f f_{1}\right)+\mathscr{D} f=0
$$

where $\mathscr{D} f$ has the same meaning as in $(2,2)$ but $J\left(f f_{1}\right)$ now stands for

$$
\iiint\left(f f_{1}-f^{\prime} f_{1}^{\prime}\right) \mathrm{g} b d b d \varepsilon d \mathbf{c}_{1}
$$

Inserting $(2,7)$ in $(2,8)$ we obtain respectively for $J\left(f f_{1}\right)$ and $\mathscr{D} f$ the following expressions

$$
\begin{aligned}
J\left(f f_{1}\right) & =\frac{1}{\theta^{2}} J\left(f^{(0)} f_{1}^{(0)}\right)+\frac{1}{\theta}\left[J\left(f^{(0)} f_{1}^{(1)}\right)+J\left(f_{1}^{(0)} f^{(1)}\right)\right]+\ldots \\
& \equiv \frac{1}{\theta^{2}} J^{(0)}+\frac{1}{\theta} J^{(1)}+\ldots
\end{aligned}
$$

where

$$
J^{(r)}=J\left(f^{(0)} f_{1}^{(r)}\right)+J\left(f^{(1)} f_{1}^{(r-1)}\right)+\ldots+J\left(f^{(r)} f_{1}^{(0)}\right)
$$


and

$$
\begin{aligned}
\mathscr{D} f & =\left(\sum_{0}^{\infty} \theta^{r} \frac{\partial_{r}}{\partial t}+\mathbf{c} \cdot \frac{\partial}{\partial \mathbf{r}}\right)\left(\frac{1}{\theta} f^{(0)}+f^{(1)}+\ldots\right) \\
& =\frac{1}{\theta} \mathscr{D}^{(1)}+\mathscr{D}^{(2)}+\theta \mathscr{D}^{(3)}+\ldots
\end{aligned}
$$

while use has been made of the fact indicated by $(2,3)$ in writing the first term of $\mathscr{D f}$ i.e. $\partial f / \partial t$ in the form

where

$$
\sum_{0}^{\infty} \theta^{r} \frac{\partial_{r} f}{\partial t}
$$

$$
\frac{\partial_{r} f}{\partial t}=\frac{\partial f}{\partial n} \frac{\partial_{r} n}{\partial t}+\frac{\partial f}{\partial \mathbf{u}} \cdot \frac{\partial_{r} \mathbf{u}}{\partial t}+\frac{\partial f}{\partial T} \frac{\partial_{r} T}{\partial t}
$$

The notations

$$
\frac{\partial_{r} n}{\partial t}, \frac{\partial_{r} \mathbf{u}}{\partial t} \text { and } \frac{\partial_{r} T}{\partial t}
$$

are explained in [1]. And further

$$
\mathscr{D}^{(r)} \equiv \frac{\partial_{0} f^{(r-1)}}{\partial t}+\frac{\partial_{1} f^{(r-2)}}{\partial t}+\ldots+\frac{\partial_{r-1} f^{(0)}}{\partial t}+\mathbf{c} \cdot \frac{\partial f^{(r-1)}}{\partial \mathbf{r}} ;(r>0),
$$

and

$$
\mathscr{D}^{(0)} \equiv 0
$$

The above division of $\mathscr{D} f$ is dictated by the fact that the conservation equations for momentum and energy giving the time derivatives of $\mathbf{u}$ and $T$ involve mean value functions i.e. pressure tensor and heat flux vector which can be evaluated only when $f$ is known. "This causes difficulty in the determination of $f$ by successive approximation, because the time derivatives of $n, \mathbf{u}$ and $T$ determine that of $f^{(0)}$, which effects the equation from which $f^{(1)}$ is determined. At this stage, however, as $f$ is only partially known, the pressure tensor and heat flux vector cannot be completely evaluated". Hence the special mode of $\mathscr{D} f$ as indicated in $(2,9)$. Thus

$$
\begin{aligned}
\xi(f) & =\mathscr{D} f+J\left(f f_{1}\right) \\
& =\frac{1}{\theta^{2}} J^{(0)}+\frac{1}{\theta}\left(J^{(1)}+\mathscr{D}^{(1)}\right)+\ldots \\
& =\frac{1}{\theta^{2}} \xi^{(0)}+\frac{1}{\theta} \xi^{(1)}+\ldots \quad\left(\text { if } \xi^{(r)}=J^{(r)}+\mathscr{D}^{(r)}\right)
\end{aligned}
$$


If $\xi^{(r)}=0$ for every $r$, then $\xi(f)$ vanishes for all values of $\theta$ which gives a set of integral equations for the functions $f^{(0)}, f^{(1)}, \ldots$. The first term $f^{(0)}$ of $f$ satisfies an equation which is identical to the one satisfied by the velocity distribution function in the uniform steady state. This amounts to the choice, as a valid first approximation to $f$ at $\mathbf{r}, t$ of the Maxwellian function corresponding to the number density, massvelocity and temperature at $\mathbf{r}, t$. In consequence $n, \mathbf{u}$ and $T$ can be computed with $f^{(0)}$ as well as with $f$ and this implies that we can require from all higher approximations $f^{(r)}(r>0)$ that

$$
\int f^{(r)} \psi^{(i)} d \mathbf{c}=0 \quad(i=1,2,3)
$$

where $\psi^{(i)}$ 's denote summational invariants.

In order to obtain the second approximation to $f$ Chapman and Cowling [1] solve the equation

$$
\xi^{(1)}=J^{(1)}+\mathscr{D}^{(1)}
$$

by writing

$$
f^{(1)}=f^{(0)} \Phi^{(1)}
$$

and expand $\Phi^{(1)}$ in an infinite series of Sonine polynomials in the argument of $\mathrm{c}^{2}$. The infinite set of coefficients in this expansion is given by the ratio of two infinite determinants neither of which is, in general, convergent. Chapman and Cowling consider only a finite number of elements in the determinants and the ratios of the determinants converge rapidly as the order of the determinants is increased.

Certain terms have been obtained by Wang Chang and Uhlenbeck for the third approximation. With each added approximation one must include more boundary conditions to solve the Boltzmann equation. A detailed discussion of boundary conditions is given by Grad [4]. Moreover, the complexity of the successive terms $f^{(r)}$ increases with $r$.

Finally, it should be remarked, however, that the method of Enskog-Chapman has very limited applicability. The same can be said about the method of Hilbert, the details of which can be found in [4].

\subsubsection{The method of models}

(i) Bhatnagar, Gross and Krook [5] proposed a model for collision processes in gases. They introduced some modifications into the collision terms of the Boltzmann equation $(2,1)$. An equation which has been suggested as a replacement for the Boltzmann equation $(2,1)$ is 


$$
\frac{\partial f}{\partial t}+\mathbf{c} \cdot \frac{\partial f}{\partial \mathbf{r}}=v\left(f^{(0)}-f\right)
$$

where $f^{(0)}$ is locally Maxwellian and $v$ is the collision frequency. If $v$ is taken to be constant, this equation implies the conservation equations and the decrease of $H$ where

$$
H=\int f \log f d \mathbf{c}
$$

Physically interpreted, $(2,15)$ expresses explicitly the fact that the particle collisions tend to relax the distribution function to that corresponding to local and ultimately absolute equilibrium.

The equation $(2,15)$ has been successfully used in a number of problems $[5,6,7$ and 8]. However, it would seem that solution of the actual Boltzmann equation $(2,1)$ in any specific problem is not very much more complicated. In cases where the requisite collision integrals have already been evaluated, there is hardly any difference at all in labour.

(ii) Glansdorff [9] introduced the idea of a two-fluid model for the shock wave. This in fact was an extension to the gas dynamics of the two-fluid model already introduced in the superfluidity theory.

The shock process is regarded as an irreversible physical reaction corresponding to the passage of molecules from a distribution (1) to a distribution (2). The moments of Glansdorff equations do not reduce to the Boltzmann transport equations for any but the collision invariants. This fact was pointed out by Ziering, Ek and Koch [10]. Further, the inelastic collision parameter and the number density are not determined in Glansdorff's analysis. Ziering et al have rectified these defects and calculated shock thickness.

\subsubsection{The method of moments}

In the method of moments the transport equations are used rather than the Boltzmann equation itself. Instead of dealing with a non-linear integro-differential equation governing the distribution function we deal with a set of first-order, non-linear differential equations governing the spatial and temporal variation of the parameter, giving the statistical characteristics of the distribution function. The number of these differential equations must equal the number of unknown statistical parameters. If $\Phi(c)$ be the some function of the molecular velocity we get after multiplying $(2,1)$ by $\Phi(\mathbf{c})$ and integrating over all values of $\mathbf{c}$ the equation

$$
\int \Phi(\mathbf{c})\left(\frac{\partial f}{\partial t}+\mathbf{c} \cdot \frac{\partial f}{\partial \mathbf{r}}\right) d \mathbf{c}=\iiint \int \Phi(\mathbf{c})\left(f^{\prime} f_{1}^{\prime}-f f_{1}\right) \mathrm{g} b d b d \varepsilon d \mathbf{c}_{1} d \mathbf{c},
$$


which is known as the Boltzmann transport equation for the function $\Phi(\mathbf{c})$. More details about Maxwell's equations of transfer are given in chapter 4 of this thesis. The method of moments then consists in assuming an explicit distribution function with the unknown parameters and performing the necessary integrations indicated in $(2,17)$ for suitably chosen functions $\Phi(\mathbf{c})$. We then obtain a set of non-linear differential equations in these parameters whose solution is then sought.

(i) Burnett $[11,12]$ expands the distribution function in a series of Sonine polynominals and solid harmonics in the form

$$
\begin{aligned}
f & =\left(\frac{m}{2 \pi k T}\right)^{\frac{3}{2}} \exp \left(-\frac{m}{2 k T} \mathrm{C}^{2}\right)\left\{\sum_{n} n ! \Gamma\left(n+\frac{3}{2}\right) A_{n} S_{\frac{1}{2}}^{n}\left(\frac{m}{2 k T} \mathrm{C}^{2}\right)+\right. \\
& \left.+\sum_{\kappa}\left(\frac{m}{2 k T}\right)^{\frac{1}{2} \kappa} \sum_{n} n ! \Gamma\left(\kappa+n+\frac{3}{2}\right) Y_{\kappa n} S_{\kappa+\frac{1}{2}}^{n}\left(\frac{m}{2 k T} \mathbf{C}^{2}\right)\right\}
\end{aligned}
$$

where $\Gamma(m+n+1) S_{m}^{n}(x)$ is the coefficient of $s^{n}$ in the expansion of $(1-s)^{-m-1}$ $\cdot e^{-x s /(1-s)}$, and $Y_{\kappa n}$ represents a solid harmonic function of $U, V, W$, of degree $\kappa$, where $(U, V, W)$ or $\mathbf{C}$ is the velocity of a molecule relative to the mean velocity of the gas as a whole. The coefficients $A_{n}, T$ and the mass velocity which are functions of $\mathbf{r}$ are determined by considering the moments of the Boltzmann equation $(2,1)$.

(ii) Grad [13] represents the distribution function $f$ as a product of the Maxwellian distribution function and an infinite series of 'generalized' Hermite polynomials in the dimensionless velocity $\mathbf{v}^{\prime}=\mathbf{v}(k T)^{-\frac{1}{2}}$ where $\mathbf{v}=\mathbf{c}-\mathbf{u}$ and $\mathbf{u}=1 / n \int \mathbf{c} f d \mathbf{c}$. Then

$$
f=f^{(0)}\left(a^{(0)} H^{(0)}+a_{i}^{(1)} H_{i}^{(1)}+\frac{1}{2 !} a_{i j}^{(2)} H_{i j}^{(2)}+\ldots\right)
$$

the first few polynomials are

$$
H^{(0)}=1 ; H_{i}^{(1)}=v_{i}^{\prime} ; H_{i j}^{(2)}=v_{i}^{\prime} v_{j}^{\prime}-\delta_{i j} \text {, etc. etc. }
$$

At equilibrium all the $a$ 's are zero except $a^{(0)}$, which represents the equilibrium spatial distribution of particles. The Hermite polynomials form an orthonormal set with the weight function $f^{(0)}$. Hence the coefficients $a_{i j} \ldots^{(m)}$ are related to $f$ through

$$
a_{i j} \cdots^{(m)}=\frac{1}{n(\mathbf{r}, t)} \int f H_{i j} \cdots^{(m)} d \mathbf{c}
$$


The first few $a$ 's are connected to the moments of $f$ by

$$
\left.\begin{array}{l}
a^{(0)}=1, \\
a_{i}^{(1)}=0 \\
a_{i j}^{(2)}=\frac{p_{i j}}{p} \\
a_{i j k}^{(3)}=\frac{S_{i j k}}{p(k T)^{\frac{1}{2}}}
\end{array}\right\}
$$

Hence in course of solving the Boltzmann equation one obtains expressions for the tensors of interest in terms of the intermolecular forces.

Grad breaks off the series for $f$ by retaining terms only upto the third Hermite polynomial. When $(2,19)$ is inserted into the Boltzmann equation with terms retained upto $\mathrm{a}^{(3)}$, and with terms quadratic in the $a$ 's neglected, a set of differential equations is obtained for the $a$ 's or for the thermodynamic quantities $\varrho, \mathbf{u}, T, p_{i j}$ and $S_{i j k}$. When he approximates $S_{i j k}$ by a linear combination of the contractions, he can discuss all quantities in terms of the thirteen moments of $f, \varrho, \mathbf{u}, T, p_{i j}$, and $S_{i}$ (the equation of state relates $\varrho$ and $p_{i i}$ ). In this manner one obtains a system of thirteen ordinary differential equations whose solution is sought numerically.

(iii) Mott-Smith [14] assumes the distribution function to be the sum of two Maxwellian terms with different temperatures and mean velocities:

with

$$
f=f_{\alpha}+f_{\beta}
$$

$$
f_{\alpha}=n_{\alpha}(x)\left(\frac{m}{2 \pi k T_{\alpha}}\right)^{\frac{3}{2}} \exp \left[-\frac{m}{2 k T_{\alpha}}\left(\mathbf{c}-\mathbf{i} u_{\alpha}\right)^{2}\right],
$$

and similarly for $f_{\beta}$ with $\alpha$ replaced by $\beta$ throughout. Here the space densities are the only undetermined parameters. Equations of conservation together with a transport equation for $u^{2}$ or $u^{3}$ are used to solve for the unknown parameters $n_{\alpha}$ and $n_{\beta}$ of the functions $f_{\alpha}$ and $f_{\beta}$ respectively. One can choose any function for $\Phi$ in the transport equation, but the simplest choice is found to be $\Phi=u^{n}$, for which the integrations can be carried out with relative ease. The calculations are carried out by Mott-Smith for $n=2$ and $n=3$.

Instead of assuming the distribution function as the sum of two Maxwellian terms as in $(2,23)$, Holway [15] introduces a new approximation procedure based on the use of ellipsoidal velocity distributions. The distribution function is supposed to be of the form 
where

$$
f(\mathbf{c}, x)=n_{1}(x) \Phi_{1}+\Phi_{2} \sum a_{n m}(x) c^{2 n} c_{1}^{m}
$$

and

$$
\Phi_{1}=\left(2 \pi R T_{\alpha}\right)^{-\frac{3}{2}} \exp \left[-\frac{1}{2 R T_{\alpha}}\left(\left(c_{1}-u_{\alpha}\right)^{2}+c_{2}^{2}+c_{3}^{2}\right)\right],
$$

$$
\Phi_{2}=(2 \pi)^{-\frac{3}{2}}\left(\lambda_{22}\right)^{-1}\left(\lambda_{11}\right)^{-\frac{1}{2}} \exp \left[-\frac{1}{2}\left(\frac{\left(c_{1}-Q\right)^{2}}{\lambda_{11}}+\frac{c_{2}^{2}+c_{3}^{2}}{\lambda_{22}}\right)\right] \text {. }
$$

The parameters $n_{1}(x), \lambda_{11}(x), \lambda_{22}(x), Q(x)$ and $a_{n m}(x)$ are all functions of position and are determined by requiring the distribution function to satisfy an appropriate number of independent transport equations. In reality Holway considers the simplest case of the approximate form given by $(2,24)$ in which the expansion coefficients $a_{n m}$ are all taken to be zero except for $a_{00}\left(=n_{2}\right)$.

On the other hand Lees [16] introduces the two stream Maxwellian which is a natural extension and generalization of Mott-Smith's function [14] for a normal shock, but differs from it in certain essential respect. One important difference between Lees' formulation and Mott-Smith's is that the distribution functions employed by Lees are discontinuous in particle velocity, whereas Mott-Smith's function is continuous. Lees applies his technique to linearized plane couette flow and Rayleigh's problem and to that end he utilizes two stream Maxwellians in equations of transfer.

By using the line of sight principle postulated by Lees [16] which in fact divides the molecules into groups as if they were in free-molecule flow, a more general formulation for the velocity distribution function (viz the bimodel two stream Maxwellian distribution) is proposed by Beck [17]. The assumption of Lees for the velocity distribution function is contained as a special case in the formulation of Beck. Whereas Shen [18] proposes a relaxation type procedure for the transition regime of rarefied gas flows and applies it to the problem of couette flow. He makes use of Lees' two stream Maxwellians in his assumed form of distribution function. This point is taken up again in chapter 4 of this thesis, where the present author proposes a new method "Modified transfer equation approach". Further Takao's work [19] is similar to that of Shen.

(iv) Ikenberry and Truesdell [20] introduce a method called "Maxwellian Iteration" for the solution of the equations of transfer. The distinguishing characteristic of this method of solving the transport equations is that no special forms or series for the distribution function need be specified. Assuming only the existence and differentiability of the moments a simple iterative scheme is constructed. The equilibrium solution is taken for the zero ${ }^{\text {th }}$ iterate; the first iterates yield the Newton-Fourier formula for the flux of energy; the second iterate for the pressures, which follows almost without additional calculation, is essentially the Burnett formula.

In summary, the method of moments leads to an infinite set of coupled equations, 
which cannot be solved in general. Thus it is necessary to cut off the equations at some points by means of more or less arbitrary assumptions about the higher moments. The fact that such an assumption must be made introduces an approximation into the theory. Thus the method of moments is, in practice, an approximate technique for solving the Boltzmann equation. However, the truncated set of equations can often be solved, so that the moment technique usually does lead to a simpler set of equations than the original Boltzmann equation.

\subsubsection{The variational method using a restricted variational principle}

Rosen [21] proposed the restricted variational principle:

$$
\lim _{\bar{f} \rightarrow f} \delta \int_{-\infty}^{+\infty} \int_{c}\left[\bar{f} u \frac{\partial f}{\partial x}+\frac{\partial_{e} \bar{f}}{\partial t} f\right] d x d \mathbf{c}=0
$$

such that $f$ is varied as a function of $x$ and $\mathbf{c}$ and $\bar{f}$ is held fixed when the variation is carried out. $\partial_{e} \bar{f} / \partial t$ is the rate of change of $\bar{f}$ caused by molecular encounters. We also take $f$ to be given at the boundaries $x= \pm \infty,|\mathbf{c}|=\infty$. The variational principle then leads to

$$
\int_{-\infty}^{+\infty} \int_{c}\left[u \frac{\partial f}{\partial x}-\frac{\partial_{e} f}{\partial t}\right] \delta f d x d \mathbf{c}=0
$$

If $\delta f$ is arbitrary, then we obtain the Boltzmann equation for a shock wave.

In any actual problem the trial solution must satisfy

$$
\int \psi^{(i)} u \frac{\partial f}{\partial x} d \mathbf{c}=0 \quad(i=1,2,3)
$$

so as to conserve mass, momentum and energy. $\psi^{(i)}$ 's in $(2,27)$ represent the collision invariants.

It is interesting to note that here again the method of solution consists in assuming an explicit distribution function with suitable parameters which may be obtained by solving the Euler equations of the variational problem. Rosen [22] assumes the MottSmith velocity distribution function $(2,23)$ for his problem.

This method did not find much applications, the reason seems to be the prohibitive work involved during the analysis.

\subsubsection{Numerical procedures}

The development of numerical techniques for solving the Boltzmann equation is still in infancy. The start seems to be encouraging but it will be long before something 
definite can be said about the numerical techniques developed so far. The principal obstacle in the way of solving the Boltzmann equation is the non-linearity and complexity of collision integral in it. Here we give a brief review of the important numerical procedures developed upto now for solving the Boltzmann equation or its imitations.

Broadwell [23] introduced the discrete ordinate method for studying the problems in rarefied gas flows. This method already existed in the field of radiative transfer and further discussion of this must be referred to the work of Chandrasekher [24]. Later, Hamel and Wachman [25] investigated this quadrature technique more fully than Broadwell did. "The numerical method developed was one of discrete ordinates and involved analysing the linearized Boltzmann equation for hard sphere molecules at discrete points in velocity space with the collision integral approximated by a finite sum through the use of Gauss quadrature formulas". This numerical method has now further been extended to cover the non-linear Boltzmann equation by Wachman and Hamel [26]. Recently, Huang and Giddens [27] have suggested a further improvement in the discrete ordinate method and on this basis they propose a new method for treating the various problems (linearized) in the kinetic theory of gases. A Monte Carlo method has been developed by Nordsieck and Hicks [28] for solving the Boltzmann equation. In particular the attention is directed on the evaluation of the nonlinear five dimensional collision integral.

\subsection{Conclusion}

Inspite of the seeming generality of most of these methods, it is interesting to see that they are in practice most suitable for certain class of problems. In order to know the relative merits of various techniques for solving the Boltzmann equation one must study the solution of a number of representative problems of kinetic theory of gases.

It is to be noted, however, that the recent trend in kinetic theory is towards the scrutiny of the distribution function itself. This trend is pursued in chapters 3-4 of this thesis where new forms of the distribution functions are suggested and applied to certain practical problems. 


\section{COLLISION OF GAS CLOUDS}

\subsection{Introduction}

Collision of gaseous columns or clouds are frequently of importance in problems of Aeronautical and Astrophysical interest. In Aeronautical applications, the interaction of a jet of gas discharging into another gas which may be either at rest or in motion arises while considering the behaviour of jet engine or rocket engine exhausts. In Astrophysical applications, problems of interaction of interstellar matter concentrated in the form of discrete clouds, which are in random motion, are of interest. Further, whereas in Aeronautical problems the gas densities involved are of order $10^{23}$ molecules/cc, in Astrophysical problems the densities are of order 10 molecules /cc. Extensive work on the discharge of gas jets has been done for several years by numerous authors using a continuum model for the gas for compressible and incompressible, laminar and turbulent jets. Extensive observational data on the interstellar medium is available in Astrophysical literature. However, theoretical studies on the collision of interstellar clouds are few [29]. Here the astrophysical problem of collision of gas clouds is investigated from molecular point of view. The clouds are supposed to be homogeneous and a Sutherland model is assumed for the molecules. Further, the clouds are supposed to consist of monatomic molecules. The present approach is inspired by the work of Mott-Smith [14] where he treats the problem of a normal shock wave.

\subsection{Formulation of the problem}

Consider two clouds, $\alpha$ and $\beta$ of gas, moving in directions opposite to each other with the respective velocities $\tilde{u}_{\alpha}$ and $\tilde{u}_{\beta}$ parallel to the x-axis. Let $f_{\alpha}$ and $f_{\beta}$ be the velocity distribution functions of the clouds $\alpha$ and $\beta$ respectively. We shall regard the resultant cloud as a homogeneous mixture of both the clouds $\alpha$ and $\beta$, whose statistical properties vary only with the time $t$. If $f_{\gamma}$ be the velocity distribution function of the resultant cloud $\gamma$ then at each moment are present gas $\alpha$, gas $\beta$, and the mixture $\gamma$ and the distribution function $f$ at any time may be regarded as

$$
f=f_{\alpha}+f_{\beta}+f_{\gamma},
$$

where

$$
f_{i}(t)=n_{i}(t)\left(\frac{m}{2 \pi k T_{i}}\right)^{\frac{3}{2}} \exp \left[-\frac{m}{2 k T_{i}}\left(\mathbf{v}-\mathbf{i} \tilde{u}_{i}\right)^{2}\right] \quad(i=\alpha, \beta, \gamma)
$$


with $n_{i}(t), T_{i}$ and $\tilde{u}_{i}$ representing, respectively, the number density, temperature and mass velocity of the cloud $i$. It is to be noted, however, that only space densities are unassigned and are assumed to be function of time only. In the absence of external forces, the distribution function $f$ is determined by the Boltzmann equation and in the notation of Chapman and Cowling [1] but with $\mathbf{c}$ replaced by $\mathbf{v}(u, v, w)$ it is written as

$$
\frac{\partial f}{\partial t}=\iint\left(f^{\prime} f_{1}^{\prime}-f f_{1}\right) k_{1} d \mathbf{k} d \mathbf{v}_{1}
$$

where only binary collisions are considered and the primed distribution functions are the final values after collision.

\subsection{Conservation equations and consistency relations}

If $\Phi(\mathbf{v})$ be some known function of $\mathbf{v}$, the corresponding transport equation is

$$
\frac{\partial}{\partial t} \int \Phi(\mathbf{v}) f(\mathbf{v}, t) d \mathbf{v}=\iiint\left(\Phi^{\prime}-\Phi\right) f f_{1} \mathrm{~g} d \Omega d \mathbf{v}_{1} d \mathbf{v}
$$

where $\mathrm{g}$ is the magnitude of the relative velocity given by

$$
\mathrm{g}=\left|\mathbf{v}_{1}-\mathbf{v}\right|=\left|\mathbf{v}_{1}{ }^{\prime}-\mathbf{v}^{\prime}\right|
$$

and $d \Omega=b d b d \varepsilon, b$ and $\varepsilon$ are defined in section 2.1. After substituting for $f$ from equation $(3,1)$, the transport equation $(3,4)$ may be written as

$$
\frac{\partial}{\partial t} \int \Phi(\mathbf{v}) f d \mathbf{v}=\sum_{i, j} I_{i j} \quad(i, j=\alpha, \beta, \gamma)
$$

where

$$
I_{i j}=\iiint\left(\Phi^{\prime}-\Phi\right) f_{i} f_{j 1} \mathrm{~g} d \Omega d \mathbf{v}_{1} d \mathbf{v}
$$

However, since the distribution functions $f_{i}$ are all assumed to be Maxwellian, we have

$$
f_{i} f_{i 1}=f_{i}^{\prime} f_{i 1}
$$

and from this, it follows [1] that

$$
I_{\alpha \alpha}=I_{\beta \beta}=I_{\gamma \gamma}=0
$$

The functions $\Phi(v)$ are chosen to ensure conservation of mass, momentum and energy during the collision of the clouds. Thus by giving $\Phi$ the values $1, u, \frac{1}{2} \mathrm{v}^{2}$, in succession, we have the following three equations of conservation 


$$
\begin{aligned}
& \frac{d n_{\alpha}}{d t}+\frac{d n_{\beta}}{d t}+\frac{d n_{\gamma}}{d t}=0 \\
& \tilde{u}_{\alpha} \frac{d n_{\alpha}}{d t}+\tilde{u}_{\beta} \frac{d n_{\beta}}{d t}+\tilde{u}_{\gamma} \frac{d n_{\gamma}}{d t}=0 \\
& \left(3 R T_{\alpha}+\tilde{u}_{\alpha}^{2}\right) \frac{d n_{\alpha}}{d t}+\left(3 R T_{\beta}+\tilde{u}_{\beta}^{2}\right) \frac{d n_{\beta}}{d t}+\left(3 R T_{\gamma}+\tilde{u}_{\gamma}^{2}\right) \frac{d n_{\gamma}}{d t}=0
\end{aligned}
$$

Eliminating $n_{\gamma}$ from the equations $(3,10),(3,11)$ and 3,12$)$, we have

$$
\begin{aligned}
& \left(\tilde{u}_{\alpha}-\tilde{u}_{\gamma}\right) \frac{d n_{\alpha}}{d t}+\left(\tilde{u}_{\beta}-\tilde{u}_{\gamma}\right) \frac{d n_{\beta}}{d t}=0 \\
& \left\{3 R\left(T_{\alpha}-T_{\gamma}\right)+\left(\tilde{u}_{\alpha}^{2}-\tilde{u}_{\gamma}^{2}\right)\right\} \frac{d n_{\alpha}}{d t}+\left\{3 R\left(T_{\beta}-T_{\gamma}\right)+\left(\tilde{u}_{\beta}^{2}-\tilde{u}_{\gamma}^{2}\right)\right\} \frac{d n_{\beta}}{d t}=0
\end{aligned}
$$

For nontrivial solutions for $\left(d n_{\alpha} / d t\right)$ and $\left(d n_{\beta} / d t\right)$ from equations $(3,13)$ and $(3,14)$ we must have

$$
\frac{3 R\left(T_{\alpha}-T_{\gamma}\right)+\left(\tilde{u}_{\alpha}^{2}-\tilde{u}_{\gamma}^{2}\right)}{3 R\left(T_{\beta}-T_{\gamma}\right)+\left(\tilde{u}_{\beta}^{2}-\tilde{u}_{\gamma}^{2}\right)}=\frac{\left(\tilde{u}_{\alpha}-\tilde{u}_{\gamma}\right)}{\left(\tilde{u}_{\beta}-\tilde{u}_{\gamma}\right)}
$$

Assuming that the two clouds are moving with equal but oppositely directed velocities $\tilde{u}_{\alpha}=-\tilde{u}_{\beta}$ and have the same temperature, it is clear that the resulting single cloud must be at rest. Thus, if we put

$$
\left.\begin{array}{l}
\tilde{u}_{\beta}=-\tilde{u}_{\alpha}, \\
T_{\alpha}=T_{\beta}, \\
\tilde{u}_{\gamma}=0
\end{array}\right\}
$$

then, equation $(3,15)$ reduces to

$$
\left(T_{\gamma}-T_{\alpha}\right)=\frac{1}{3}\left(\frac{\tilde{u}_{\alpha}^{2}}{R}\right)
$$

which is the temperature rise of the clouds due to collision. 


\subsection{Transport equation}

By virtue of equation $(3,15)$, the pair of equations $(3,13)$ and $(3,14)$ reduce to a single differential equation for the two unknowns $n_{\alpha}$ and $n_{\beta}$. Therefore, one more equation is necessary to solve for the unknowns. This is provided by an additional transport equation for which we take $\Phi(\mathbf{v})=u^{2}$ and we have

$$
\frac{\partial}{\partial t} \int u^{2} f(\mathbf{v}, t) d \mathbf{v}=\iiint \int\left(u^{\prime 2}-u^{2}\right) f f_{1} \mathbf{g} b d b d \varepsilon d \mathbf{v}_{1} d \mathbf{v}
$$

For the evaluation of the right hand side of equation $(3,18)$ we first consider the integral

$$
\int_{0}^{\infty} \int_{0}^{2 \pi}\left(u^{\prime 2}-u^{2}\right) \mathrm{g} b d b d \varepsilon
$$

Let us define the vector

$$
\mathbf{K}=\mathbf{v}^{\prime}-\mathbf{v}=\mathbf{v}_{1}-\mathbf{v}_{\mathbf{1}}^{\prime}
$$

And let $\psi$ be the angle between $\mathbf{g}$ and $\mathbf{K}, \theta$ the angle between $\mathbf{g}$ and $\mathbf{i}$, the direction of the flow of the gas clouds. Further, let $\varepsilon$ be the angle between the plane of $\mathbf{g}$ and $\mathbf{K}$ and the plane of $\mathbf{g}$ and $\mathbf{i}$. Then from a well known formula of spherical trigonometry, we have

$$
\cos (\mathbf{v}, \mathbf{g})=\cos \theta \cos \psi+\sin \theta \sin \psi \cos \varepsilon
$$

so that

$$
u^{\prime}-u=\mathrm{K}(\cos \theta \cos \psi+\sin \theta \sin \psi \cos \varepsilon)
$$

where

$$
\mathrm{K}=\mathrm{g} \cos \psi \text { and also } u_{1}-u=\mathrm{g} \cos \theta
$$

Also, the angle $\chi$ through which the initial relative velocity is deflected is given by the collision laws to be

$$
\psi=\frac{1}{2}(\pi-\chi)
$$

Integrating with respect to $\varepsilon$ and making use of equations $(3,22)$ and $(3,24)$, the integral $(3,19)$ transforms to the following expression

$$
\begin{gathered}
\int_{0}^{\infty} \int_{0}^{2 \pi}\left(u^{\prime 2}-u^{2}\right) \mathrm{g} b d b d \varepsilon=2 \pi \int_{0}^{\infty}\left[\left(u_{1}-u\right)^{2} \cos ^{4} \psi+2 u\left(u_{1}-u\right) \cos ^{2} \psi+\right. \\
\left.+\frac{1}{2}\left\{\mathrm{~g}^{2}-\left(u_{1}-u\right)^{2} \sin ^{2} \psi \cos ^{2} \psi\right\}\right] \mathrm{g} b d b
\end{gathered}
$$


Interchanging $u_{1}$ and $u$ in $(3,25)$ and adding the result to $(3,25)$ we obtain

where

$$
2 \pi \int_{0}^{\infty}\left[\mathrm{g}^{2}-3\left(u_{1}-u\right)^{2}\right] \sin ^{2} \psi \cos ^{2} \psi \mathrm{g} b d b=\frac{\pi}{2} \Phi_{11}^{(2)}(\mathrm{g})\left[\mathrm{g}^{2}-3\left(u_{1}-u\right)^{2}\right],
$$

$$
\Phi_{11}^{(l)}(\mathrm{g})=\int\left(1-\cos ^{l} \chi\right) \mathrm{g} b d b
$$

Therefore, combining equation $(3,7)$ with the equations $(3,2)$ and $(3,26)$, we have

$$
\begin{aligned}
& I_{\beta \alpha}+I_{\alpha \beta}=\frac{1}{2} \pi n_{\alpha} n_{\beta}\left(\frac{m}{2 \pi k T_{\alpha}}\right)^{3} \iint \Phi_{11}^{(2)}(\mathrm{g})\left[\mathrm{g}^{2}-3\left(u_{1}-u\right)^{2}\right] \times \\
& \times \exp \left\{-\frac{m}{2 k T_{\alpha}}\left[\left(\mathbf{v}-\mathbf{i} \tilde{u}_{\alpha}\right)^{2}+\left(\mathbf{v}_{1}+\mathbf{i} \tilde{u}_{\alpha}\right)^{2}\right]\right\} d \mathbf{v}_{1} d \mathbf{v}
\end{aligned}
$$

Putting

$$
\left.\begin{array}{l}
\mathscr{C}_{\alpha}=\left(\frac{m}{2 k T_{\alpha}}\right)^{\frac{1}{2}}\left(\mathbf{v}-\mathbf{i} \tilde{u}_{\alpha}\right), \\
\mathscr{C}_{\beta}=\left(\frac{m}{2 k T_{\beta}}\right)^{\frac{1}{2}}\left(\mathbf{v}_{1}+\mathbf{i} \tilde{u}_{\alpha}\right)
\end{array}\right\}
$$

and defining two new vectors $\Lambda(\lambda, \mu, v)$ and $\Lambda_{1}\left(\lambda_{1}, \mu_{1}, v_{1}\right)$ by

$$
\left.\begin{array}{l}
\mathscr{C}_{\alpha}=\Lambda-\frac{1}{2} \boldsymbol{\Lambda}_{1} \\
\mathscr{C}_{\beta}=\boldsymbol{\Lambda}+\frac{1}{2} \boldsymbol{\Lambda}_{1}
\end{array}\right\}
$$

and integrating over $\boldsymbol{\Lambda}$, equation $(3,28)$ becomes

$$
\begin{aligned}
& I_{\alpha \beta}+I_{\beta \alpha}=\frac{1}{4}(2 \pi)^{-\frac{1}{2}} n_{\alpha} n_{\beta} \int \Phi_{11}^{(2)}(\mathrm{g})\left\{\left(\frac{2 k T_{\alpha}}{m}\right) \Lambda_{1}^{2}-4\left(\frac{2 k T_{\alpha}}{m}\right)^{\frac{1}{2}} \tilde{u}_{\alpha} \lambda_{1}+\right. \\
& \left.+4 \tilde{u}_{\alpha}^{2}-3\left[\left(\frac{2 k T_{\alpha}}{m}\right)^{\frac{1}{2}} \lambda_{1}-4 \tilde{u}_{\alpha}^{2}\right]\right\} \exp \left(-\frac{1}{2} \Lambda_{1}^{2}\right) d \Lambda_{1}
\end{aligned}
$$

Introducing spherical coordinates for $\boldsymbol{\Lambda}_{1}\left(\Lambda_{1}, \theta_{1}, \varphi_{1}\right)$ such that

$$
\left.\begin{array}{l}
\lambda_{1}=\Lambda_{1} \cos \theta_{1} ; \mu_{1}^{2}+v_{1}{ }^{2}=\Lambda_{1}{ }^{2} \sin ^{2} \theta_{1} \\
0<\Lambda_{1}<\infty ; 0 \leqslant \varphi_{1} \leqslant 2 \pi ; 0 \leqslant \theta_{1} \leqslant \pi
\end{array}\right\}
$$

and integrating over $\varphi_{1}$, equation $(3,31)$ gives 


$$
\begin{aligned}
& I_{\alpha \beta}+I_{\beta \alpha}=\left(\frac{4}{\pi}\right)\left(\frac{m}{k T_{\alpha}}\right)^{\frac{3}{2}} n_{\alpha} n_{\beta} \tilde{u}_{\alpha}^{5} \iint \Phi_{11}^{(2)}(\mathrm{g}) \times \\
& \times\left[\Lambda_{0}^{2}-2 \Lambda_{0} \cos \theta_{1}+1-3\left(\Lambda_{0} \cos \theta_{1}-1\right)^{2}\right] \exp \left[-\frac{m}{k T_{\alpha}} \tilde{u}_{\alpha}^{2} \Lambda_{0}^{2}\right] \times \\
& \times \Lambda_{0}^{2} \sin \theta_{1} d \theta_{1} d \Lambda_{0}
\end{aligned}
$$

where

$$
\Lambda_{0}=\frac{1}{2}\left(\frac{2 k T_{\alpha}}{m}\right)^{\frac{1}{2}}\left(\frac{\Lambda_{1}}{\tilde{u}_{\alpha}}\right)
$$

we make a further transformation

$$
r^{2}=\Lambda_{0}^{2}-2 \Lambda_{0} \cos \theta_{1}+1
$$

with limits of integration for $r$ as $\left|1+\Lambda_{0}\right|$ and $\left|1-\Lambda_{0}\right|$ corresponding, respectively, to $\theta_{1}=\pi$ and $\theta_{1}=0$. Furthermore to complete the integration in $(3,33)$, we choose the law of interaction of the molecules to be given by the Sutherland law

$$
\Phi_{11}^{(2)}(\mathrm{g})=\frac{1}{3} \mathrm{~g} \sigma^{2}+\left(\frac{k s}{m}\right)\left(\frac{\sigma^{2}}{\mathrm{~g}}\right)
$$

where $s$ is Sutherland's constant having the dimensions of temperature and $\sigma$ is the molecular diameter. Substituting $(3,36)$ in $(3,33)$ and integrating over $\theta_{1}$ and $\Lambda_{0}$ we have

where

$$
I_{\alpha \beta}+I_{\beta \alpha}=\left(\frac{\pi^{\frac{1}{2}}}{6}\right) \sigma^{2} \tilde{u}_{\alpha} n_{\alpha} n_{\beta}\left[4 \tilde{u}_{\alpha}^{2} A_{\alpha \beta}(t)-\left(\frac{k s}{m}\right) A_{s_{\alpha \beta}}(t)\right],
$$

$$
\begin{aligned}
& A_{\alpha \beta}(t)=4 \pi^{\frac{1}{2}}\left(1+\frac{3}{2 t^{2}}-\frac{3}{4 t^{4}}+\frac{3}{8 t^{6}}\right) \operatorname{erf} t+4\left(1+\frac{1}{t^{2}}-\frac{3}{4 t^{4}}\right) \frac{e^{-t^{2}}}{t} \\
& A_{s_{\alpha \beta}}(t)=\left(\frac{12}{t}-\frac{18}{t^{2}}-\frac{1}{t^{3}}-\frac{101}{15} t+\frac{128}{15} t^{2}\right) e^{-t^{2}}-\pi^{\frac{1}{2}}\left(\frac{9}{t^{2}}+\frac{1}{2}\right) \operatorname{erf} t \\
& \operatorname{erf} t=\frac{2}{\sqrt{ } \pi} \int_{0}^{\infty} e^{-x^{2}} d x
\end{aligned}
$$

and

$$
t=\left(\frac{m}{k T_{\alpha}}\right)^{\frac{1}{2}} \tilde{u}_{\alpha}
$$

All the other collision integrals may be calculated in a similar manner. Hence the transport equation $(3,18)$ finally becomes 


$$
\begin{aligned}
{\left[R\left(T_{\alpha}-T_{\gamma}\right)\right.} & \left.+\tilde{u}_{\alpha}^{2}\right]\left[\frac{d n_{\alpha}}{d t}+\frac{d n_{\beta}}{d t}\right]= \\
& -\frac{\pi^{\frac{1}{2}}}{6} \sigma^{2} \tilde{u}_{\alpha} n_{\alpha} n_{\beta}\left[-4 \tilde{u}_{\alpha}^{2} A_{\alpha \beta}(t)+\left(\frac{k s}{m}\right) A_{s_{\alpha \beta}}(t)\right]- \\
& -\frac{\pi^{\frac{1}{2}}}{6} \sigma^{2} \tilde{u}_{\alpha} n_{\beta} n_{\gamma}\left[-\tilde{u}_{\alpha}^{2} A_{\beta \gamma}(t)+\left(\frac{k s}{m}\right) A_{s_{\alpha \beta}}(t)\right]- \\
& -\frac{\pi^{\frac{1}{2}}}{6} \sigma^{2} \tilde{u}_{\alpha} n_{\alpha} n_{\gamma}\left[-\tilde{u}_{\alpha}^{2} A_{\gamma \alpha}(t)+\left(\frac{k s}{m}\right) A_{s_{\gamma \alpha}}(t)\right]
\end{aligned}
$$

\subsection{Nondimensionalization}

Since the total number of molecules of all species is constant, we have

$$
n_{\alpha}+n_{\beta}+n_{\gamma}=n_{\alpha 0}+n_{\beta 0}
$$

where the suffix 0 denotes the inital value of the quantity suffixed at $t=0$, the equations $(3,11)$ and $(3,42)$ can be written, respectively, by eliminating $n_{\gamma}$, in the dimensionless form

$$
\frac{d v_{\alpha}}{d \tau}-\frac{d v_{\beta}}{d \tau}=0
$$

$\frac{d v_{\alpha}}{d \tau}+\frac{d v_{\beta}}{d \tau}=v_{\alpha}^{2} g_{\gamma \alpha}+v_{\beta}^{2} g_{\beta \gamma}+v_{\alpha} v_{\beta}\left(g_{\alpha \beta}+g_{\beta \gamma}+g_{\gamma \alpha}\right)-v_{\alpha}\left(1+v_{0}\right) g_{\gamma \alpha}-v_{\beta}\left(1+v_{0}\right) g_{\beta \gamma}$

where

$$
\begin{gathered}
\tau=\frac{t}{\tau_{1}} ; \quad v_{\alpha}=\frac{n_{\alpha}}{n_{\alpha 0}} ; \quad v_{\beta}=\frac{n_{\beta}}{n_{\alpha 0}} ; \quad v_{0}=\frac{n_{\beta 0}}{n_{\alpha 0}}, \\
\tau_{1}=\text { mean collision time }=\frac{1}{4}\left(\pi R T_{\alpha}\right)^{-\frac{1}{2}} \sigma^{-2} n_{\alpha 0}^{-1}, \\
g_{\alpha \beta}=\left[-4 \tilde{u}_{\alpha}^{2} A_{\alpha \beta}(t)+\left(\frac{k s}{m}\right) A_{s_{\alpha \beta}}(t)\right] / 16 \tilde{u}_{\alpha}\left(R T_{\alpha}\right)^{\frac{1}{2}}, \\
g_{\beta \gamma}=\left[-\tilde{u}_{\alpha}^{2} A_{\beta \gamma}(t)+\left(\frac{k s}{m}\right) A_{s_{\beta \gamma}}(t)\right] / 32 \tilde{u}_{\alpha}\left(R T_{\alpha}\right)^{\frac{1}{2}}, \\
g_{\gamma \alpha}=\left[-\tilde{u}_{\alpha}^{2} A_{\gamma \alpha}(t)+\left(\frac{k s}{m}\right) A_{s_{\gamma \alpha}}(t)\right] / 32 \tilde{u}_{\alpha}\left(R T_{\alpha}\right)^{\frac{1}{2}}
\end{gathered}
$$




\subsection{Solution and discussion}

In order to determine the unknowns $v_{\alpha}$ and $v_{\beta}$, we have to solve the pair of equations $(3,44)$ and $(3,45)$. Regarding $\left(v_{\alpha}+v_{\beta}\right)$ and $\left(v_{\alpha}-v_{\beta}\right)$ as the new unknowns, equations $(3,44)$ and $(3,45)$ can be written respectively as

$$
\begin{aligned}
& \frac{d\left(v_{\alpha}-v_{\beta}\right)}{d \tau}=0, \\
& \frac{d\left(v_{\alpha}+v_{\beta}\right)}{d \tau}=A\left(v_{\alpha}+v_{\beta}\right)^{2}+B\left(v_{\alpha}+v_{\beta}\right)+C,
\end{aligned}
$$

where

and

$$
\begin{aligned}
& A=\frac{1}{2}\left(g_{\gamma \alpha}+g_{\beta \gamma}+\frac{1}{2} g_{\alpha \beta}\right), \\
& B=-\left(g_{\beta \gamma}+v_{0} g_{\gamma \alpha}\right),
\end{aligned}
$$

$$
C=-\left(1-v_{0}\right)\left[\frac{1}{4}\left(1-v_{0}\right) g_{\alpha \beta}+\left(1+v_{0}\right)\left(g_{\beta \gamma}-g_{\gamma \alpha}\right)\right]
$$

In writing $(3,45)$ in the form $(3,47)$ the use has been made of the following result obtained from $(3,46)$ i.e.

$$
v_{\alpha}-v_{\beta}=\text { constant }=v_{\alpha 0}-v_{\beta 0}=1-v_{0} .
$$

Now the solution of the equation $(3,47)$ is given by

$$
v_{\alpha}+v_{\beta}=\frac{\left[D\left(B+q^{\frac{1}{2}}\right) e^{-\tau q^{\frac{1}{2}}}-\left(B-q^{\frac{1}{2}}\right)\right]}{2 A\left(1-D e^{-\tau q^{\frac{1}{2}}}\right)},
$$

where

$$
\begin{aligned}
D & =\frac{2 A\left(1+v_{0}\right)+B-q^{\frac{1}{2}}}{2 A\left(1+v_{0}\right)+B+q^{\frac{1}{2}}} \\
q & =\frac{1}{4}\left(1-v_{0}^{2}\right) g_{\alpha \beta}^{2}+\left(3-2 v_{0}^{2}\right) g_{\beta \gamma}^{2}-\left(2-3 v_{0}^{2}\right) g_{\gamma \alpha}^{2}+2 v_{0} g_{\beta \gamma} g_{\gamma \alpha}+ \\
& +\frac{1}{2}\left(1-v_{0}\right)\left(3+v_{0}\right) g_{\alpha \beta} g_{\beta \gamma}-\frac{1}{2}\left(1-v_{0}\right)\left(1+3 v_{0}\right) g_{\alpha \beta} g_{\gamma \alpha}
\end{aligned}
$$

This completes the solution of the problem. $v_{\alpha}$ and $v_{\beta}$ can readily be calculated from $(3,51)$ and $(3,52)$.

In the particular case when the initial densities of the clouds $n_{\alpha 0}$ and $n_{\beta 0}$ are equal, then $v_{0}=1$ and the solution simplifies to

$$
v_{\alpha}=v_{\beta}=\frac{B_{1} D_{1} e^{-B_{1} \tau}}{2 A_{1}\left(1-D_{1} e^{-B_{1} \tau}\right)}
$$


and

$$
v_{y}=\frac{2\left(1-e^{-B_{1} \tau}\right)}{\left(1-D_{1} e^{-B_{1} \tau}\right)}
$$

with

$$
\begin{aligned}
& A_{1}=\frac{1}{2}\left(g_{\gamma \alpha}+g_{\beta \gamma}+\frac{1}{2} g_{\alpha \beta}\right), \\
& B_{1}=-\left(g_{\beta \gamma}+g_{\gamma \alpha}\right),
\end{aligned}
$$

$$
D_{1}=\frac{2 A}{A+B}
$$

It is seen from $(3,56)$ that $v_{\gamma} \rightarrow 2$ as $\tau \rightarrow \infty$.

\subsection{Concluding remarks}

Once the densities are determined, the complete distribution function is known and a knowledge of the Boltzmann distribution function is sufficient to determine the local macroscopic properties. It is conjectured, however, that the approach developed above for the gas clouds may be useful in solving the problems of the kinetic theory of the reacting gases. 


\section{Part I Modified transfer equation approach}

\subsection{Introduction}

While dealing with the problems in rarefied gases, it is desirable to find the direct solution of the Boltzmann equation for the single particle distribution function in accordance with prescribed boundary conditions. However, the mathematical complexity of the problem is such that one is led to look for alternatives to make some headway. Among others one of the powerful tools is transfer equation approach and through this approach many practical problems in the theory of rarefied gases become susceptible to mathematical treatment. In this approach the Maxwell Boltzmann equation is satisfied in a certain average sense, rather than at every point. This procedure hinges on the assumed form of the velocity distribution function which contains a number of coefficients with spatial and temporal variations and these coefficients are determined by satisfying judiciously selected transfer equations as indicated in section 2.2.3. Major contributions for the advancement of the transfer equation approach have been made by Mott-Smith [14], Lees [16], Shen [18] and Beck [17]. These methods have been dealt with in section 2.2.3.

In this chapter a new and a more general formulation for the velocity distribution function is proposed and the main features of the present method viz. modified transfer equation approach are exposed in sections 4.2 and 4.3. In part II of this chapter the proposed method is applied to linearized couette flow.

\subsection{Preliminary remarks prior to the introduction of the present method}

As has been remarked earlier, Shen utilizes Lees' two stream Maxwellians in his assumed form of the distribution function. This point is taken up at the appropriate place again in section 4.3. The present author believes that instead of using Lees' twostream Maxwellian distributions it is more amenable to use polynomials in the velocity space. In Shen's approach one can only choose 'appropriate' transfer equations so as to calculate the unknowns introduced in the distribution function and this introduces an undesirable rigidity into the procedure. In the present analysis we endeavour to remove this restriction and here one can choose as many transfer equations as one requires. Moreover, the series expansion of the distribution function has more variables which are functions of position and can be evaluated by the 
appropriate (unrestricted) number of transfer equations whereas in Shen's approach there are only two unknowns. Therefore the present method is more general than that of Shen's and can take full advantage of the additional transfer equations.

\subsection{Basic theory of the present method}

One of the ways of understanding the problems related to the Boltzmann equation is to construct model equations that are easier to solve and in which one or the other characteristic of the equation is emphasized. One such model has been postulated by Bhatnagar, Gross and Krook [5] and Welander [30]. This model (known as BGK model) provides the basis for the assumption of the distribution function and which in turn forms the starting point in the present approach. This model equation is written as

$$
\left(\frac{\partial}{\partial t}+\mathbf{v} \cdot \nabla\right) n f=-v\left(n f-n^{(0)} f^{(0)}\right)
$$

where

$$
\begin{aligned}
& f=f(t, \mathbf{r}, \mathbf{v}), \text { velocity distribution function } \\
& \mathbf{v}=\left(v_{x}, v_{y}, v_{z}\right) \text {, molecular velocity } \\
& n^{(0)} f^{(0)}=n(\mathbf{r}, t)\left[\frac{m}{2 \pi k T(\mathbf{r}, t)}\right]^{\frac{3}{2}} \exp \left[-\frac{m}{2 k T(\mathbf{r}, t)(}(\mathbf{v}-\mathbf{q}(\mathbf{r}, t))^{2}\right], \\
& n(\mathbf{r}, t)=\int f d \mathbf{v}, \quad \text { number density } \\
& \mathbf{q}(\mathbf{r}, t)=n^{-1} \int \mathbf{v} f d \mathbf{v}, \quad \text { macroscopic velocity } \\
& T(\mathbf{r}, t)=\frac{m}{3 n(\mathbf{r}, t) k} \int f(\mathbf{v}-\mathbf{q}(\mathbf{r}, t))^{2} d \mathbf{v}, \quad \text { temperature }
\end{aligned}
$$

and $v$ is the collision frequency.

The BGK model is based on the assumption that the molecules scattered into the beam of molecules come from collision between molecules into Maxwellian motion. This model equation exhibits all the elementary qualitative features of the Boltzmann equation.

Under steady state conditions

$$
\frac{\partial f}{\partial t}=0
$$


and assuming $n^{(0)} f^{(0)}$ to be constant, hence upon integration equation $(4,1)$ results as

$$
n f=n^{\prime} f^{\prime} \exp \left[-v\left(\mathrm{~s}-\mathrm{s}^{\prime}\right) / \mathrm{v}\right]+n^{(0)} f^{(0)}\left[1-\exp \left\{-v\left(\mathrm{~s}-\mathrm{s}^{\prime}\right) / \mathrm{v}\right\}\right]
$$

where $s, \mathrm{v}, n^{\prime} f^{\prime}$ represent the distance measured along the direction of $\mathbf{v}$, the magnitude of the velocity $\mathbf{v}$ and the distribution prescribed at the place of origin $\left(s=s^{\prime}\right)$ of the molecules having velocity $\mathbf{v}$ respectively. In his paper Shen proposes an approximate distribution function which formally resembles $(4,3)$, viz.

$$
n f=n^{\prime} f^{\prime} \exp \left[-v\left(s-s^{\prime}\right) / \mathrm{v}\right]+n^{*} f^{*}\left[1-\exp \left\{-v\left(\mathrm{~s}-\mathrm{s}^{\prime}\right) / \mathrm{v}\right\}\right]
$$

where the distribution function $n^{*} f^{*}$ is not the same as the local Maxwellian $n^{(0)} f^{(0)}$.

The extent of manoeuvrability in the selection of $n^{*} f^{*}$ is aggrandized by the arbitrariness of the function itself but the choice is to be guided by a practical view point only rather than the aesthetic sense. Shen employs Lees' type of distribution for $n^{*} f^{*}$ and this choice brings in its trail an undesirable rigidity which makes the procedure rather restrictive because mainly of the following fact: "The function $n^{*} f$ " has a very limited number of constants which precludes the full exploitation of the transfer equations".

Therefore it becomes expedient to introduce the series type expansion for $n^{*} f^{*}$ with as many unknowns as possible which can be evaluated with the aid of transfer equations. The above procedure is applied to linearized couette flow and the analysis is given in the following section.

\section{Part II Modified transfer equation method for linearized plane couette flow}

\subsection{Formulation of the problem}

Problems, to which a kinetic theory of gases is adequately applicable, are practically limited to a few cases, in which deviations from equilibrium are small, or a simple form of the collision integral can be postulated. Thus the testbed of any proposed method lies in its applicability to some well known problems. Here the present approach is applied to the problem of steady flow of gas between two parallel plates.

The upper plate moves with velocity $+u / 2$ in its own plane at $y=d / 2$, while the lower plate at $y=-d / 2$ moves parallel to the upper plate with velocity $-u / 2$. In the following analysis we assume $d=2$ for the sake of simplicity. Both the temperature and the density $\varrho$ (hence the number density $n$ ) are constants for the linearized case.

Further it is necessary to select a model for the interaction of the molecules with these plates, and for simplicity it is assumed that the molecules reflect with Maxwell equilibrium distribution specified by the plate temperature, i.e. diffuse reflection with complete thermal accommodation. Hence on leaving the plates $y= \pm 1$, the molecules have the Maxwellian distribution 


$$
n f=n A \exp \left\{-h\left[\left(v_{x} \mp \frac{u}{2}\right)^{2}+v_{y}^{2}+v_{z}^{2}\right]\right\}
$$

with

$$
A=\left(\frac{\pi}{h}\right)^{-\frac{3}{2}}
$$

and

$$
h=\frac{1}{2 R T}
$$

The upper sign in $(4,5)$ holds for plate ' 1 ' while the lower sign for plate ' 2 '. The figure is shown below.

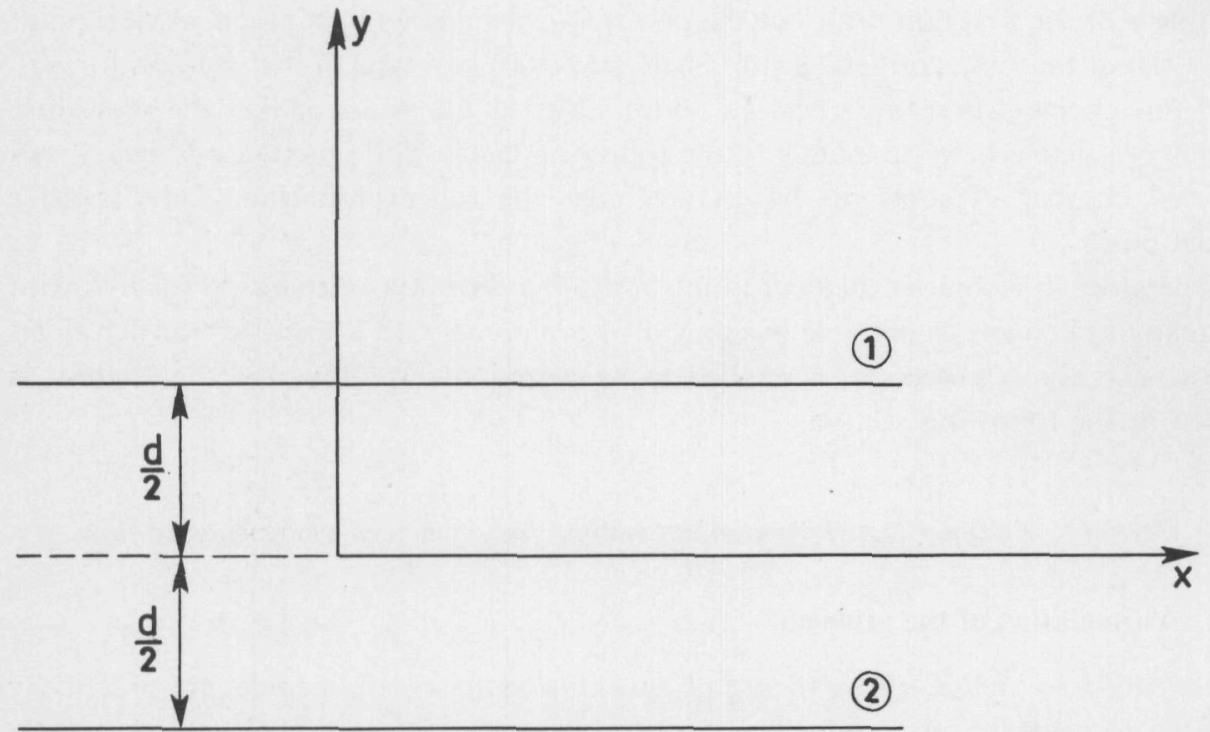

Fig. 1

\subsection{Choice of the distribution function}

The only independent variable in this problem is the coordinate normal to the plate; thus $(4,4)$ transforms to

$$
n f=n^{\prime} f^{\prime} \exp \left[-\frac{v\left(y-y^{\prime}\right)}{v_{y}}\right]+n^{*} f^{*}\left[1-\exp \left\{-\frac{v\left(y-y^{\prime}\right)}{v_{y}}\right\}\right]
$$

where $v_{y}$ is the component of molecular velocity in the $y$-direction and $y^{\prime}$ indicates the place of origin of the molecules. 
As the choice of $n^{*} f^{*}$ is arbitrary, in the present problem we assume the distribution function for $n^{*} f^{*}$ a series expansion in velocity space as follows:

$$
\begin{aligned}
& v_{y}>0, \\
& n f=n A \exp \left\{-h\left[\left(v_{x}+\frac{u}{2}\right)^{2}+v_{y}^{2}+v_{z}^{2}\right]-\frac{v(y+1)}{v_{y}}\right\}+ \\
& +n A\left[1-\exp \left(-\frac{v(y+1)}{v_{y}}\right)\right] \exp \left\{-h v^{2}\right\}\left[\alpha_{1}^{+}(y) v_{x}+\alpha_{2}^{+}(y) v_{y}+\alpha_{3}^{+}(y) v_{x} v_{z}+\ldots\right]
\end{aligned}
$$

$v_{y}<0$,

$$
\begin{aligned}
& n f=n A \exp \left\{-h\left[\left(v_{x}-\frac{u}{2}\right)^{2}+v_{y}^{2}+v_{z}^{2}\right]-\frac{v(y-1)}{v_{y}}\right\}+ \\
& +n A\left[1-\exp \left(-\frac{v(y-1)}{v_{y}}\right)\right] \exp \left\{-h \mathrm{v}^{2}\right\}\left[\alpha_{1}^{-}(y) v_{x}+\alpha_{2}^{-}(y) v_{y}+\ldots\right]
\end{aligned}
$$

where $\alpha_{i}^{\mp}(y)$ are functions of $y$ only and are to be determined by means of transfer equations. Further, the boundary conditions are automatically satisfied by $(4,9)$.

\subsection{Calculation of the coefficients $\alpha_{i}^{\mp}(y)$}

In order to calculate $\alpha_{i}^{\mp}(y)$ we make use of the transfer equations and first consider the equations for $m v_{x}$ and $m v_{x} v_{y}$. It is worth remarking that while considering the transfer equations for the above said variables all the terms except with coefficients $\alpha_{1}^{\mp}(y)$ vanish as a result of the form of the distribution function $(4,9)$. Moreover, if we choose other transfer equations e.g. for $m v_{y}$ and $m v_{y}^{2}$, the same sort of simplification is bound to result. It is thus evident that on account of the broad choice of transfer equations, one may be able to span the wider spectrum of non-linear problems. Without any loss of generality, we will calculate only four of the variables introduced in the distribution functions and make use of four moment equations in order to calculate them.

\subsubsection{Maxwell's equation of transfer}

We wish to seek for the general equation for the rate of transport of $\Phi$ which is any function of the velocity component of the particle. Assuming that there is no external field of force acting on the molecules, the changes in $\Phi$ can then be of two sorts only:

1. The influx and efflux of $\Phi$ arising from molecular transport of $\Phi$ through the bounding surfaces of the element, and

2. The changes in $\Phi$ produced by molecular collisions inside the element. 
These changes are given [16] respectively by

and

$$
-\nabla_{r} \cdot\left[\int f \mathbf{v} \Phi d \mathbf{v}\right]
$$

$$
\iiint \int\left(\Phi^{\prime}-\Phi\right) f f_{1} \mathrm{~g} b d b d \varepsilon d \mathbf{v} d \mathbf{v}_{1}
$$

where the symbols have the usual meaning. Further the total amount of $\Phi$ per unit volume is given by

with

$$
n \bar{\Phi}=\int \Phi f d \mathbf{v},
$$

$$
n=\int f d \mathbf{v}
$$

and $\Phi$ denotes mean or expected value of $\Phi$. The rate of increase of this amount per unit time is

$$
\frac{\partial}{\partial t}(n \bar{\Phi})
$$

and this must be equal to the sum of the changes noted above. Hence, the Maxwell's integral equation of transfer is

$$
\frac{\partial}{\partial t} \int f \Phi d \mathbf{v}+\nabla_{r} \cdot\left[\int f \mathbf{v} \Phi d \mathbf{v}\right]=\iiint \int\left(\Phi^{\prime}-\Phi\right) f f_{1} \mathbf{g} b d b d \varepsilon d \mathbf{v} d \mathbf{v}_{1}
$$

For the steady case, $(4,15)$ reduces to

$$
\nabla_{r} \cdot\left[\int f \mathbf{v} \Phi d \mathbf{v}\right]=\iiint \int\left(\Phi^{\prime}-\Phi\right) f f_{1} \mathrm{~g} b d b d \varepsilon d \mathbf{v} d \mathbf{v}_{1},
$$

which in the notation of Chapman and Cowling can be written as [1]

$$
\nabla_{r} \cdot\left[\int f \mathbf{v} \Phi d \mathbf{v}\right]=\int \Phi\left(\frac{\partial f}{\partial t}\right)_{\text {coll }} d \mathbf{v}=n \Delta \Phi
$$

As was shown by Maxwell [31], the ordinary gas dynamic conservation equations are obtained regardless of the choice of $f$ by taking $\Phi$ to be successively the summational or collisional invariants of mass, momentum and energy i.e.

$$
\Phi=m, m \mathbf{v}, \frac{m v^{2}}{2}
$$

for which

$$
\Delta \bar{\Phi}=0
$$


and the number of additional moments employed depends on the degree of detail required.

Maxwell [31] also proved that it is not necessary at all to introduce the actual expression for the law of distribution into the right hand side of equation $(4,17)$ if we restrict ourselves to a particular law of force (repulsive field force $\sim r^{-5}$ ). In other words, for Maxwellian molecules the right hand side of equation $(4,17)$ is independent of the form of the distribution function and then it can be expressed in terms of macroscopic flow quantities.

\subsubsection{Calculation of $\alpha_{1}^{\mp}(y)$}

For the calculation of $\alpha_{1}^{\mp}(y)$ we consider the transfer equations for $m v_{x}$ and $m v_{x} v_{y}$. Taking cognizance of the equations $(4,17)$ and $(4,18)$ the transfer equations for $m v_{x}$ and $m v_{x} v_{y}$, respectively, are

$$
\begin{aligned}
& \frac{d}{d y} \int f m v_{x} v_{y} d \mathbf{v}=0 \\
& \frac{d}{d y} \int f m v_{x} v_{y}^{2} d \mathbf{v}=\frac{R T}{\mu} p_{x y}
\end{aligned}
$$

where the integration in $(4,20)$ and $(4,21)$ stands for the following notation

$$
\int[\ldots] f d \mathbf{v}=\int_{-\infty}^{+\infty} \int_{0}^{+\infty} \int_{-\infty}^{+\infty}[\ldots] f_{v_{y}>0} d v_{x} d v_{y} d v_{z}+\int_{-\infty}^{+\infty} \int_{-\infty}^{0} \int_{-\infty}^{+\infty}[\ldots] f_{v_{y}<0} d v_{x} d v_{y} d v_{z}
$$

with $f_{v_{y}>0}$ and $f_{v_{y}<0}$ representing, respectively, the distribution function for $v_{y}>0$ and $v_{y}<0$. It is to be remarked, however, that individually the functions $f_{v_{y}>0}$ and $f_{v_{y}<0}$ do not have any real meaning but only when taken as a totality they represent the distribution function.

Inserting the expression $(4,9)$ for the distribution function in the equations $(4,20)$ and $(4,21)$ we obtain the following equations for $\alpha_{1}^{\mp}(y)$

$$
\begin{aligned}
& \frac{\alpha_{1}^{+}}{4 \sqrt{\pi} h^{\frac{3}{2}}}\left(1-G_{1,+}\right)-\frac{\alpha_{1}^{-}}{4 \sqrt{\pi} h^{\frac{3}{2}}}\left(1-G_{1,-}\right)=-\frac{1}{\varrho} p_{x y}+\frac{u}{4 \sqrt{\pi h}}\left(G_{1,+}+G_{1,-}\right) \\
& \frac{\alpha_{1}^{+}}{8 h^{2}}\left(1-G_{2,+}\right)+\frac{\alpha_{1}^{-}}{8 h^{2}}\left(1-G_{2,-}\right)=\frac{R T}{\mu} p_{x y}\left(y+c_{1}\right)+\frac{u}{8 h}\left(G_{2,+}-G_{2,-}\right)
\end{aligned}
$$

where

$$
G_{n, \pm}=G_{n}[v \sqrt{ } h(1 \pm y)]
$$


and $G_{n}(\alpha)$ denotes the function defined by

$$
\left.G_{n} \alpha\right)=\frac{\int_{0}^{\infty} \exp \left(-x^{2}-\alpha / x\right) x^{n} d x}{\int_{0}^{\infty} \exp \left(-x^{2}\right) x^{n} d x}, \quad(n=0,1,2, \ldots)
$$

with $\alpha$ representing a real and positive number. This function occurs not only in the kinetic theory of gases but also in other domains of theoretical physics. It has been studied analytically by Abramowitz [32] and numerically by Welander [30], and Huang [33].

Before determining $\alpha_{1}^{\mp}(y)$ we must first determine the constants $p_{x y}$ and $c_{1}$ which occur in the equations $(4,23)$ and $(4,24)$. These constants are evaluated with the aid of 'end' conditions

$$
y= \pm 1
$$

It is interesting to note that the equations for $\alpha_{1}^{\mp}(y)$ turn out to be of a similar nature as those for $u_{ \pm}$in case of Shen. In other words we must be able to get Shen's results when we consider only the first term in the series expansion for the velocity distribution function $(4,9)$.

It is easy to see from $(4,25)$ and $(4,26)$ that the following relations hold

$$
\begin{aligned}
& G_{n,+}=G_{n}[2 v \sqrt{ } h] ; \quad G_{n,-}=G_{n}[0]=1 \text { for } y=+1, \\
& G_{n,+}=G_{n}[0]=1 ; \quad G_{n,-}=G_{n}[2 v \sqrt{ } h] \text { for } y=-1
\end{aligned}
$$

Substituting $(4,27)$ in equations $(4,23)$ and $(4,24)$, and making use of the relations $(4,28)$ and $(4,29)$ we obtain the following equations

$$
\begin{gathered}
\frac{\alpha_{1}^{+}}{4 \sqrt{\pi} h^{\frac{3}{2}}}\left(1-G_{1}^{+}\right)=-\frac{1}{\varrho} p_{x y}+\frac{u}{4 \sqrt{\pi h}}\left(G_{1}^{+}+1\right) \\
\frac{\alpha_{1}^{+}}{8 h^{2}}\left(1-G_{2}^{+}\right)=\frac{R T}{\mu} p_{x y}\left(1+c_{1}\right)+\frac{u}{8 h}\left(G_{2}^{+}-1\right) \\
-\frac{\alpha_{1}^{-}}{4 \sqrt{\pi} h^{\frac{3}{2}}}\left(1-G_{1}^{+}\right)=-\frac{1}{\varrho} p_{x y}+\frac{u}{4 \sqrt{\pi h}}\left(1+G_{1}^{+}\right) \\
\frac{\alpha_{1}^{-}}{8 h^{2}}\left(1-G_{2}^{+}\right)=\frac{R T}{\mu} p_{x y}\left(-1+c_{1}\right)+\frac{u}{8 h}\left(1-G_{2}^{+}\right)
\end{gathered}
$$

where the symbols $G_{1}{ }^{+}, G_{2}{ }^{+}$stand for $G_{1}[2 v \sqrt{ } h]$ and $G_{2}[2 v \sqrt{ } h]$ respectively. 
From $(4,30)$ and $(4,31)$ it follows that

$$
\frac{1-G_{1}^{+}}{1-G_{2}^{+}}=\frac{1}{2} \sqrt{\frac{\pi}{h}} \cdot \frac{-\frac{1}{\varrho} p_{x y}+\frac{u}{4 \sqrt{\pi h}}\left(1+G_{1}^{+}\right)}{\frac{R T}{\mu} p_{x y}\left(1+c_{1}\right)+\frac{u}{8 h}\left(G_{2}^{+}-1\right)}
$$

The expression for the ratio $\left(1-G_{1}{ }^{+}\right) /\left(1-G_{2}{ }^{+}\right)$can also be obtained from the equations $(4,32)$ and $(4,33)$ but the expression so obtained differs from that given by $(4,34)$ and which is

$$
\frac{1-G_{1}^{+}}{1-G_{2}^{+}}=-\frac{1}{2} \sqrt{\frac{\pi}{h}} \cdot \frac{-\frac{1}{\varrho} p_{x y}+\frac{u}{4 \sqrt{\pi h}}\left(1+G_{1}^{+}\right)}{\frac{R T}{\mu} p_{x y}\left(-1+c_{1}\right)+\frac{u}{8 h}\left(1-G_{2}^{+}\right)}
$$

on comparing $(4,34)$ and $(4,35)$ we conclude that following relations must hold

$$
\begin{aligned}
& c_{1}=0 \\
& p_{x y}=\frac{u}{2 \sqrt{h \pi}} /\left\{\frac{1}{\varrho}+\frac{R T}{\mu} \frac{2 \sqrt{ } h}{\sqrt{ } \pi} \frac{1-G_{1}^{+}}{1-G_{2}^{+}}\right\}
\end{aligned}
$$

once $c_{1}, p_{x y}$ are evaluated, $\alpha_{1}^{\mp}$ can be readily obtained from $(4,23)$ and $(4,24)$.

As anticipated, the results given by first term in the series expansion for the distribution function $(4,9)$ are identical with those of Shen.

Now with the aid of $(4,37)$ we obtain

$$
\frac{1}{C_{D} M}=\sqrt{\frac{\pi \gamma}{2}}+\frac{1}{2} \frac{R_{e}}{M}\left(1+\frac{G_{2}^{+}-G_{1}^{+}}{1-G_{2}^{+}}\right)
$$

where

$$
\left.\begin{array}{rl}
C_{D} & =p_{x y} / \frac{1}{2} \varrho u^{2} \\
R_{e} & =(\varrho u b) / \mu \\
M & =u / \sqrt{\gamma R T}
\end{array}\right\}
$$

Thus the drag coefficient has the correct free-molecule flow value in the limit $\left(R_{e} / M\right) \rightarrow 0$ and the correct Navier-Stokes behaviour $\left(C_{D}=2 / R_{e}\right)$ in the limit $\left(R_{e} / M\right) \rightarrow \infty$. 


\subsubsection{Calculation of $\alpha_{2}^{\mp}(y)$}

In order to determine $\alpha_{2}^{\mp}(y)$ we employ the transfer equations for $m v_{y}$ and $m v_{y}{ }^{2}$. Keeping in view the equations $(4,17),(4,18),(4,19)$, and the result of Maxwell mentioned at the end of section 4.6.1, and the expression $(4,9)$ for the distribution function, the transfer equations for $m v_{y}$ and $m v_{y}{ }^{2}$ turn out, respectively, to be

$$
\begin{array}{r}
\frac{\alpha_{2}^{+}}{2 \sqrt{\pi} h^{\frac{3}{2}}}\left(1-G_{3,+}\right)-\frac{\alpha_{2}^{-}}{2 \sqrt{\pi} h^{\frac{3}{2}}}\left(1-G_{3,-}\right)=-\frac{p_{y y}}{\varrho}-\frac{1}{4 h}\left(G_{2,+}+G_{2,-}\right) \\
\frac{3 \alpha_{2}^{+}}{8 h^{2}}\left(1-G_{4,+}\right)+\frac{3 \alpha_{2}^{-}}{8 h^{2}}\left(1-G_{4,-}\right)=\frac{R T}{\mu} p_{y y}\left(y+c_{2}\right)+\frac{1}{2 \sqrt{\pi} h^{\frac{3}{2}}}\left(G_{3,-}-G_{3,+}\right)
\end{array}
$$

wheres $G$ 's have the same meaning as defined in $(4,25)$ and $(4,26)$. Substitution of $(4,27)$ in $(4,40)$ and $(4,41)$ yields the following equations

$$
\begin{aligned}
& \frac{\alpha_{2}^{+}}{2 \sqrt{\pi} h^{\frac{3}{2}}}\left(1-G_{3}^{+}\right)=-\frac{1}{\varrho} p_{y y}-\frac{1}{4 h}\left(G_{2}^{+}+1\right) \\
& \frac{3 \alpha_{2}^{+}}{8 h^{2}}\left(1-G_{4}^{+}\right)=\frac{R T}{\mu} p_{y y}\left(1+c_{2}\right)+\frac{1}{2 \sqrt{\pi} h^{\frac{3}{2}}}\left(1-G_{3}^{+}\right) \\
& -\frac{\alpha_{2}^{-}}{2 \sqrt{\pi} h^{\frac{3}{2}}}\left(1-G_{3}^{+}\right)=-\frac{1}{\varrho} p_{y y}-\frac{1}{4 h}\left(1+G_{2}^{+}\right) \\
& \frac{3 \alpha_{2}^{-}}{8 h^{2}}\left(1-G_{4}^{+}\right)=\frac{R T}{\mu} p_{y y}\left(-1+c_{2}\right)+\frac{1}{2 \sqrt{\pi} h^{\frac{3}{2}}}\left(G_{3}^{+}-1\right)
\end{aligned}
$$

where the symbols $G_{3}{ }^{+}$and $G_{4}{ }^{+}$stand for $G_{3}[2 v \sqrt{ } h]$ and $G_{4}[2 v \sqrt{ } h]$ respectively.

Now carrying out the similar analysis as has been done in section 4.6 .2 we arrive at the following relations

$$
\begin{aligned}
& c_{2}=0 \\
& p_{y y}=\frac{-\frac{1+G_{2}^{+}}{4 h}-\frac{2}{3 \pi h} \frac{\left(1-G_{3}^{+}\right)^{2}}{1-G_{4}^{+}}}{\frac{1}{\varrho}+\frac{R T}{\mu} \frac{4}{3} \sqrt{\frac{h}{\pi}} \cdot \frac{1-G_{3}^{+}}{1-G_{4}^{+}}}
\end{aligned}
$$

Once the constants $c_{2}$ and $p_{y y}$ are evaluated, we can easily determine $\alpha_{2}^{\mp}$ from $(4,40)$ and $(4,41)$. 
The constant $p_{y y}$ as given by $(4,47)$ depends on the Mach number and the Knudsen number. This can be seen by writing $(4,47)$ in the form

$$
p_{y y}=\frac{-\frac{1+G_{2}^{+}}{2}-\frac{4}{3} \frac{\left(1-G_{3}^{+}\right)^{2}}{1-G_{4}^{+}}}{M^{2}\left[\gamma+\frac{R_{e}}{M} / \frac{\gamma}{2 \pi} \cdot \frac{4}{3} \cdot \frac{1-G_{3}^{+}}{1-G_{4}^{+}}\right]} \cdot u^{2} \varrho
$$

Further we can express $p_{y y}$ in terms of $p_{x y}$ with the aid of $(4,38)$ and $(4,39)$. The relation would be of the form $p_{y y}=$ (constant). $p_{x y}$ where the (constant) is a function of Mach number and Knudsen number. The coefficients $\alpha_{2}^{\mp}$ are given by the expressions

$$
\begin{aligned}
& \alpha_{2}^{+}=\frac{\left|\begin{array}{ll}
2 \sqrt{\pi} h^{\frac{3}{2}}\left\{-p_{y y}-\frac{1}{4 h}\left(G_{2,+}+G_{2,-}\right)\right\} & -\left(1-G_{3,-}\right) \\
\frac{8}{3} h^{2}\left\{\frac{R T}{\mu} y p_{y y}+\frac{1}{2 \pi h^{\frac{3}{2}}}\left(G_{3,-}-G_{3,+}\right)\right\} & \left(1-G_{4,-}\right)
\end{array}\right|}{\left|\begin{array}{cc}
1-G_{3,+} & -\left(1-G_{3,-}\right) \\
1-G_{4,+} & 1-G_{4,-}
\end{array}\right|} \\
& \alpha_{2}^{-}=\frac{\left|\begin{array}{ll}
1-G_{3,+} & \left\{-p_{y y}-\frac{1}{4 h}\left(G_{2,+}+G_{2,-}\right)\right\} 2 \sqrt{\pi} h^{\frac{3}{2}} \\
1-G_{4,+} & \left\{\frac{R T}{\mu} y p_{y y}+\frac{1}{2 \pi h^{\frac{3}{2}}}\left(G_{3,-}-G_{3,+}\right)\right\} \frac{8}{3} h^{2}
\end{array}\right|}{\left|\begin{array}{ll}
1-G_{3,+} & -\left(1-G_{3,-}\right) \\
1-G_{4,+} & 1-G_{4,-}
\end{array}\right|}
\end{aligned}
$$

where $p_{y y}$ is given by $(4,47)$.

The dependence of $\alpha_{2}^{\mp}$ on the Mach number and the Knudsen number is via the expression of $p_{y y}$ as given in $(4,48)$. In a similar manner the other coefficients can be evaluated by choosing the suitable transfer equations. The method involves more and more labour as one goes to the higher order moments. The coefficients show explicit dependence on Mach number and Knudsen number. Thus the evaluation of the coefficients completes the determination of $(4,9)$ under the required boundary conditions. 
Chapter 5

KINETIC THEORY OF SHOCK STRUCTURE

\subsection{Introduction}

In the present age of sonic booms and nuclear explosions even the layman is familiar with the 'Shock Waves'. The shock wave is the most important gas dynamic discontinuity across which there are abrupt changes in the physical conditions (viz. velocity, temperature, etc. etc.) of a moving fluid. Such abrupt changes can occur only when flow velocities exceed the acoustic velocity and hence are characteristic of supersonic flows. The actual zone within which transition from one physical state to a second takes place is finite but extremely narrow (of the order of several mean free paths). The process or mechanism that results in this transition is very complex from a physical as well as mathematical point of view.

In this chapter we are concerned with the structure of the shock wave. The term 'structure' as applied to gas dynamic discontinuity refers to the values of the physical properties of the fluid within the small but finite thickness of the discontinuity. Moreover, the problem of shock wave structure is not beset by the mysteries of gas-solid interactions on a molecular scale. Thus surface effects such as slip do not give rise to any sort of complications.

Considerable effort has been expended in recent years in an endeavour to understand the shock structure problem and surveys concerning the shock structure problem can be found in [34], [35] and [36]. Much progress in this direction has been made in the past few years and the account is given in references [37] through [41]. In general there have been two trends in this direction. Firstly, the Boltzmann equation is reduced to the equations of macroscopic variables for general gas dynamics by the method of iteration initiated by Maxwell and these equations are applied to shock waves under special conditions. Secondly, the method consists in seeking a special function which may imply the main characteristics of the distribution within a shock wave. The unknown variables involved in this function are determined so that the function will satisfy equations of moments of the same number as that of the unknown variables. In finding such a function 'the weapon seems to be a complex of knowledge accumulated by experiences, which might be called intuition'.

In the present work is introduced a new approximation procedure based on the use of small perturbation techniques for the solution of the Krook model equation in case of shock structure problem. The main features of the Krook model are discussed in (2.2.2). The molecules of the gas are considered to be monatomic but with no internal degrees of freedom. The influence of external forces on the distribution function is neglected. The method is applicable only in case of weak shocks. The calculations are simple although quite tedious and cumbersome. 


\subsection{Basic equation and relations}

In the absence of external force the Krook model equation is written as

$$
\frac{\partial f}{\partial t}+\mathbf{v} \cdot \frac{\partial f}{\partial \mathbf{r}}=A n(F-f)
$$

where $f(\mathbf{r}, \mathbf{v}, t)$ is the distribution function and $\mathbf{v}, \mathbf{r}$ and $t$ are the molecular velocity vector, the space vector and time respectively. The number $A$ is a free parameter which in general may depend on the state of the gas.

The function $F$ in equation $(5,1)$ is the locally Maxwellian distribution function represented by

$$
F=n\left(\frac{m}{2 \pi k T}\right)^{\frac{3}{2}} \exp \left[-\frac{m}{2 k T}(\mathbf{v}-\mathbf{u})^{2}\right]
$$

where $\mathbf{u}$ is the mass velocity of the flow and $m, k$ and $T$ are mass of the particle, the Boltzmann constant and the absolute temperature respectively. Here $n, \mathbf{u}$ and $T$ are, in general, functions of $\mathbf{r}$ and $t$, and are given by

$$
\begin{aligned}
& n=\int f d \mathbf{v}, \\
& \mathbf{u}=\frac{1}{n} \int \mathbf{v} f d \mathbf{v}, \\
& \frac{3}{2 \beta}=\frac{3 k T}{m}=\frac{1}{n} \int f(\mathbf{v}-\mathbf{u})^{2} d \mathbf{v}
\end{aligned}
$$

where the integrals are evaluated over the full range of the molecular velocities.

We take shock-fixed coordinates with the velocity $u$ in the positive $x$-direction (see figure shown below).

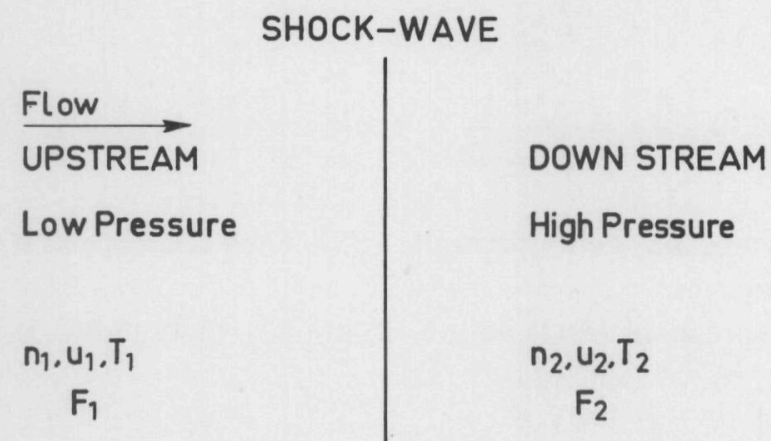

Fig. 2

$x$-axis 
The state ahead of the shock wave, i.e. for $x \rightarrow-\infty$ will be denoted by (1), the downstream state $x \rightarrow+\infty$ by (2), viz.

and

$$
n(-\infty)=n_{1}, u(-\infty)=u_{1} \text {, etc. etc. }
$$

$$
n(+\infty)=n_{2}, u(+\infty)=u_{2} \text {, etc. etc. }
$$

Equation $(5,1)$ for a steady plane normal shock can be written as

$$
v_{x} \frac{d f}{d x}=A n(F-f)
$$

where $v_{x}$ is the $x$-component of the molecular velocity vector.

It has been shown previously [42] that the equation $(5,8)$ may be integrated formally for $v_{x} \gtrless 0$, subject to the boundary conditions

$$
\begin{aligned}
& f(-\infty ; \mathbf{v})=F_{1}=n_{1}\left(\frac{\beta_{1}}{\pi}\right)^{\frac{3}{2}} \exp \left[-\beta_{1}\left(\mathbf{v}-\mathbf{u}_{1}\right)^{2}\right] \\
& f(+\infty ; \mathbf{v})=F_{2}=n_{2}\left(\frac{\beta_{2}}{\pi}\right)^{\frac{3}{2}} \exp \left[-\beta_{2}\left(\mathbf{v}-\mathbf{u}_{2}\right)^{2}\right]
\end{aligned}
$$

where the parameters are determined from the Rankine-Hugoniot conditions, to yield the expressions for the half-range distribution functions i.e.,

$$
\begin{aligned}
& f\left(x ; v_{x}>0\right)=f_{+}=\int_{-\infty}^{x} \frac{A n F}{v_{x}}\left[\exp \left(-\int_{x^{\prime}}^{x} \frac{A n}{v_{x}} d x^{\prime \prime}\right)\right] d x^{\prime}, \\
& f\left(x ; v_{x}<0\right)=f_{-}=\int_{+\infty}^{x} \frac{A n F}{v_{x}}\left[\exp \left(-\int_{x^{\prime}}^{x} \frac{A n}{v_{x}} d x^{\prime \prime}\right)\right] d x^{\prime}
\end{aligned}
$$

The rest of the details may be found in the above-cited reference as it is not necessary to repeat them here.

Inserting the expressions $(5,11)$ and $(5,12)$ in $(5,3),(5,4)$ and $(5,5)$ we obtain

$$
\begin{aligned}
n(x)= & A\left(\frac{m}{2 k \pi}\right)^{\frac{3}{2}}\left[\int_{-\infty}^{x} \frac{n^{2}}{T^{\frac{3}{2}}} \int_{0}^{\infty} \frac{1}{v_{x}} \exp \left\{-\int_{x^{\prime}}^{x} \frac{A n}{v_{x}} d x^{\prime \prime}-\frac{m}{2 k T}(\mathbf{v}-\mathbf{u})^{2}\right\}+\right. \\
& \left.+\int_{x}^{\infty} \frac{n^{2}}{T^{\frac{3}{2}}} \int_{0}^{\infty} \frac{1}{v_{x}} \exp \left\{-\int_{x^{\prime}}^{x} \frac{A n}{v_{x}} d x^{\prime \prime}-\frac{m}{2 k T}(\mathbf{v}-\mathbf{u})^{2}\right\}\right] d \mathbf{v} d x^{\prime},
\end{aligned}
$$




$$
\begin{aligned}
n(x) u(x) & =A\left(\frac{m}{2 k \pi}\right)^{\frac{3}{2}}\left[\int_{-\infty}^{x} \frac{n^{2}}{T^{\frac{3}{2}}} \int_{0}^{\infty} \frac{1}{v_{x}} \exp \left\{-\int_{x^{\prime}}^{x} \frac{A n}{v_{x}} d x^{\prime \prime}-\frac{m}{2 k T}(\mathbf{v}-\mathbf{u})^{2}\right\}+\right. \\
& \left.+\int_{x}^{\infty} \frac{n^{2}}{T^{\frac{3}{2}}} \int_{0}^{\infty} \frac{1}{v_{x}} \exp \left\{-\int_{x^{\prime}}^{x} \frac{A n}{v_{x}} d x^{\prime \prime}-\frac{m}{2 k T}(\mathbf{v}-\mathbf{u})^{2}\right\}\right] \mathbf{v} d \mathbf{v} d x^{\prime} \\
\frac{3 k}{m} \cdot n(x) T(x)= & A\left(\frac{m}{2 k \pi}\right)^{\frac{3}{2}}\left[\int_{-\infty}^{x} \frac{n^{2}}{T^{\frac{3}{2}}} \int_{0}^{\infty} \frac{1}{v_{x}} \exp \left\{-\int_{x^{\prime}}^{x} \frac{A n}{v_{x}} d x^{\prime \prime}-\frac{m}{2 k T}(\mathbf{v}-\mathbf{u})^{2}\right\}+\right. \\
& \left.+\int_{x}^{\infty} \frac{n^{2}}{T^{\frac{3}{2}}} \int_{0}^{\infty} \frac{1}{v_{x}} \exp \left\{-\int_{x^{\prime}}^{x} \frac{A n}{v_{x}} d x^{\prime \prime}-\frac{m}{2 k T}(\mathbf{v}-\mathbf{u})^{2}\right\}\right](\mathbf{v}-\mathbf{u})^{2} d \mathbf{v} d x^{\prime}
\end{aligned}
$$

Integration of the equations $(5,13),(5,14)$ and $(5,15)$ with regard to $v_{y}$ and $v_{z}$ yields

$$
\begin{aligned}
n(x)= & A\left(\frac{m}{2 k \pi}\right)^{\frac{1}{2}}\left[\int_{-\infty}^{x} \frac{n^{2}}{T^{\frac{1}{2}}} \int_{0}^{\infty} \frac{1}{v_{x}} \exp \left\{-\int_{x^{\prime}}^{x} \frac{A n}{v_{x}} d x^{\prime \prime}-\frac{m}{2 k T}\left(v_{x}-u\right)^{2}\right\}+\right. \\
& \left.+\int_{x}^{\infty} \frac{n^{2}}{T^{\frac{1}{2}}} \int_{0}^{\infty} \frac{1}{v_{x}} \exp \left\{-\int_{x^{\prime}}^{x} \frac{A n}{v_{x}} d x^{\prime \prime}-\frac{m}{2 k T}\left(v_{x}-u\right)^{2}\right\}\right] d v_{x} d x^{\prime} \\
n(x) u(x)= & A\left(\frac{m}{2 k \pi}\right)^{\frac{1}{2}}\left[\int_{-\infty}^{x} \frac{n^{2}}{T^{\frac{1}{2}}} \int_{0}^{\infty} \exp \left\{-\int_{x^{\prime}}^{x} \frac{A n}{v_{x}} d x^{\prime \prime}-\frac{m}{2 k T}\left(v_{x}-u\right)^{2}\right\}+\right. \\
& \left.+\int_{x}^{\infty} \frac{n^{2}}{T^{\frac{1}{2}}} \int_{0}^{\infty} \exp \left\{-\int_{x^{\prime}}^{x} \frac{A n}{v_{x}} d x^{\prime \prime}-\frac{m}{2 k T}\left(v_{x}-u\right)^{2}\right\}\right] d v_{x} d x^{\prime}, \\
n(x) T(x) & =\frac{2 A}{3}\left(\frac{m}{2 k \pi}\right)^{\frac{3}{2}}\left[\int_{-\infty}^{x} n^{2} T^{\frac{1}{2}} \int_{0}^{\infty} \frac{1}{v_{x}} \exp \left\{-\int_{x^{\prime}}^{x} \frac{A n}{v_{x}} d x^{\prime \prime}-\frac{m}{2 k T}\left(v_{x}-u\right)^{2}\right\}+\right. \\
& \left.+\int_{x}^{\infty} n^{2} T^{\frac{1}{2}} \int_{0}^{\infty} \frac{1}{v_{x}} \exp \left\{-\int_{x^{\prime}}^{x} \frac{A n}{v_{x}} d x^{\prime \prime}-\frac{m}{2 k T}\left(v_{x}-u\right)^{2}\right\}\right] d v_{x} d x^{\prime}+ \\
& +\frac{2 A}{3 \sqrt{ } \pi}\left(\frac{m}{2 k}\right)^{\frac{3}{2}}\left[\int_{-\infty}^{x} n^{2}(T)^{-\frac{1}{2}} \int_{0}^{\infty} \exp \left\{-\int_{x^{\prime}}^{x} \frac{A n}{v_{x}} d x^{\prime \prime}-\frac{m}{2 k T}\left(v_{x}-u\right)^{2}\right\}+\right. \\
& \left.+\int_{x}^{\infty} n^{2}(T)^{-\frac{1}{2}} \int_{0}^{\infty} \exp \left\{-\int_{x^{\prime}}^{x} \frac{A n}{v_{x}} d x^{\prime \prime}-\frac{m}{2 k T}\left(v_{x}-u\right)^{2}\right\}\right] v_{x} d v_{x} d x^{\prime}-\frac{m n(x) u^{2}}{3 k}
\end{aligned}
$$

Equations $(5,16),(5,17)$ and $(5,18)$ represent a set of singular, non-linear integral equations. 'Conservation of mass' relation is used to express $n$ in terms of $u$ and to eliminate one of the integral equations. In the following section a small perturbation technique for solving the system $(5,16), 5,17)$ and $(5,18)$ is developed. 


\subsection{General method of solution}

The disturbance number density, temperature and velocity $v, \tau$ and $\xi$ are introduced in a manner similar to that of Takano [43], but with suitable modifications as follows (see appendix $\mathrm{A})$ :

$$
\left.\begin{array}{l}
n=n_{0}(1-\varepsilon v) \\
T=T_{0}[1+\varepsilon(1-\gamma) \tau], \\
u=u_{0}(1+\varepsilon \xi)
\end{array}\right\}
$$

where $n_{0}, T_{0}, u_{0}$ are some reference number density, temperature and mass velocity which may be chosen as

$$
\left.\begin{array}{l}
n_{0}=\frac{1}{2}\left(n_{1}+n_{2}\right) \\
T_{0}=\frac{1}{2}\left(T_{1}+T_{2}\right) \\
u_{0}=\frac{1}{2}\left(u_{1}+u_{2}\right)
\end{array}\right\}
$$

where $n_{1}, n_{2}, u_{1}, u_{2}, T_{1}$ and $T_{2}$ have the same meaning as shown in the figure.

Further the parameter $\varepsilon$ appearing in $(5,19)$ is 'very' small for a weak shock and is written as

$$
\varepsilon=\frac{u_{1}-u_{2}}{u_{1}+u_{2}}
$$

We take this from of $\varepsilon$ as our small expansion parameter. In fact there exist other possible small parameters besides $\varepsilon$ but the specific choice of $\varepsilon$ as defined by $(5,21)$ turns out to be convenient for our purpose.

The order of magnitude of $v, \tau$ and $\xi$ can be deduced from $(5,19)$ by taking into account the shock conditions given by $(5,6)$ and $(5,7)$. The result is

$$
\begin{aligned}
& |v| \leqq 1, \\
& \text { and } \quad|\tau| \leqq 1, \\
& |\xi| \leqq 1 .
\end{aligned}
$$

Now we expand $v, \tau$ and $\xi$ in powers of $\varepsilon$ as 


$$
\begin{aligned}
& v=\sum_{n=0}^{\infty} v_{n}(\bar{x}) \varepsilon^{n} \\
& \tau=\sum_{n=0}^{\infty} \tau_{n}(\bar{x}) \varepsilon^{n} \\
& \xi=\sum_{n=0}^{\infty} \xi_{n}(\bar{x}) \varepsilon^{n}
\end{aligned}
$$

Where $n$ is summed up and should not be confused with ' $n$ ' denoting the density of the gas. Further $x=x_{0}+\bar{x} / \varepsilon$. This stretching of coordinates is necessitated on account of the occurrence of 'steep' gradients across the shock. Our method consists of expanding the equations $(5,16),(5,17)$ and $(5,18)$ in powers of $\varepsilon$ and from these expansions we get the governing equations for $v_{0}, \tau_{0}$ and $\xi_{0}$; the solution of which will be sought.

Left-hand sides of equations $(5,16),(5,17)$ and $(5,18)$ are given, respectively, in terms of $\varepsilon$ as follows:

$$
\begin{aligned}
n & =n_{0}(1-\varepsilon v) \\
n u & =n_{0} u_{0}\left[1+\varepsilon(\xi-v)-\varepsilon^{2} \xi v\right]
\end{aligned}
$$

and

$$
n T=n_{0} T_{0}\left[1+\varepsilon\{(1-\gamma) \tau-v\}-\varepsilon^{2}(1-\gamma) v \tau\right]
$$

It is fit to remark here that in the following analysis the integral equation $(5,16)$ is not used and instead we take advantage of the remark made at the end of the section 5.2.

Expressing the right-hand sides of the equations $(5,17)$ and $(5,18)$ in powers of $\varepsilon$ and taking into account the respective expressions $(5,27)$ and $(5,28)$ for the left-hand sides we get respectively

$$
\begin{aligned}
1+\varepsilon \xi- & \varepsilon v-\varepsilon^{2} \xi v=\frac{A n_{0}}{u_{0}}\left(\frac{m}{2 k \pi T_{0}}\right)^{\frac{1}{2}}\left[\int_{-\infty}^{\bar{x}} \int_{0}^{\infty} \exp \left\{-\frac{A n_{0}}{\varepsilon v_{x}}\left(\bar{x}-\bar{x}^{\prime}\right)-\frac{m\left(v_{x}-u_{0}\right)^{2}}{2 k T_{0}}\right\} \times\right. \\
& \times\left\{1+\varepsilon L_{1}+\varepsilon^{2} L_{2}+\ldots\right\} \frac{d \bar{x}^{\prime} d v_{x}}{\varepsilon}-\int_{\bar{x}}^{\infty} \int_{0}^{\infty} \exp \left\{\frac{A n_{0}}{\varepsilon v_{x}}\left(\bar{x}-\bar{x}^{\prime}\right)-\frac{m\left(v_{x}+u_{0}\right)^{2}}{2 k T_{0}}\right\} \times \\
& \left.\times\{\text { similar expression }\} \frac{d \bar{x}^{\prime} d v_{x}}{\varepsilon}\right]
\end{aligned}
$$




$$
\begin{aligned}
& 1+\frac{m u_{0}^{2}}{3 k T_{0}}+ \\
& +\varepsilon\left[(1-\gamma) \tau+\frac{2 m u_{0}^{2}}{3 k T_{0}} \xi-v-\frac{m u_{0}^{2}}{3 k T_{0}}\right]+\varepsilon^{2}\left[\frac{m u_{0}^{2}}{3 k T_{0}} \xi^{2}-\frac{2 m u_{0}^{2}}{3 k T_{0}} \xi \nu-(1-\gamma) v \tau\right]= \\
& =\frac{m A n_{0}}{3 k T_{0}}\left(\frac{m}{2 k \pi T_{0}}\right)^{\frac{1}{2}}\left[\int_{-\infty}^{\bar{x}} \int_{0}^{\infty} \exp \left\{-\frac{A n_{0}}{\varepsilon v_{x}}\left(\bar{x}-\bar{x}^{\prime}\right)-\frac{m\left(v_{x}-u_{0}\right)^{2}}{2 k T_{0}}\right\} \times\right. \\
& \times\left\{1+\varepsilon L_{1}+\varepsilon^{2} L_{2}+\ldots\right\} \frac{v_{x} d \bar{x}^{\prime} d v_{x}}{\varepsilon}+\int_{\bar{x}}^{\infty} \int_{0}^{\infty} \exp \left\{\frac{A n_{0}}{\varepsilon v_{x}}\left(\bar{x}-\bar{x}^{\prime}\right)-\frac{m\left(v_{x}+u_{0}\right)}{2 k T_{0}}\right\} \times \\
& \left.\times\{\text { similar terms }\} \frac{v_{x} d \bar{x}^{\prime} d v_{x}}{\varepsilon}\right]+\frac{2 A n_{0}}{3}\left(\frac{m}{2 k \pi T_{0}}\right)^{\frac{1}{2}} \times \\
& \times\left[\int_{-\infty}^{\bar{x}} \int_{0}^{\infty} \exp \left\{-\frac{A n_{0}}{\varepsilon v_{x}}\left(\bar{x}-\bar{x}^{\prime}\right)-\frac{m\left(v_{x}-u_{0}\right)^{2}}{2 k T_{0}}\right\} \times\right. \\
& \times\left\{1+\varepsilon L_{3}+\varepsilon^{2} L_{4}+\ldots\right\} \frac{d \bar{x}^{\prime} d v_{x}}{\varepsilon v_{x}}+\int_{\bar{x}}^{\infty} \int_{0}^{\infty} \exp \left\{\frac{A n_{0}}{\varepsilon v_{x}}\left(\bar{x}-\bar{x}^{\prime}\right)-\frac{m\left(v_{x}+u_{0}\right)^{2}}{2 k T_{0}}\right\} \times \\
& \left.\times\{\text { similar terms }\} \frac{d \bar{x}^{\prime} d v_{x}}{\varepsilon v_{x}}\right] \text {, }
\end{aligned}
$$

where $L_{1}, L_{2}, L_{3}$ and $L_{4}$ etc. etc. are functions of the variables $\xi, v, \tau$ and $\int_{\bar{x}^{\prime}}^{\bar{x}} v d x^{\prime \prime}$. Moreover, the occurrence of these variables in the above-mentioned functions is of the same order as that of $\varepsilon$ suffixed before that respective function. The expressions for $L_{1}$ and $L_{2}$ are given in appendix B.

Integrating the equations $(5,29)$ and $(5,30)$ by parts (see appendix B) and after some tedious calculations equations $(5,29)$ and $(5,30)$ give rise, respectively, to the following equations

$$
\begin{aligned}
& 2 \gamma \frac{d \xi}{d x}-(1-\gamma) \frac{d v}{d x}+(1-\gamma) \frac{d \tau}{d x}=\frac{u_{0} \varepsilon}{A n_{0}}\left[3(\gamma+1) \frac{d^{2} \xi}{d x^{2}}-(\gamma+3) \frac{d^{2} v}{d x^{2}}+3(1-\gamma) \frac{d^{2} \tau}{d x^{2}}\right]+ \\
&+\varepsilon\left[(1-\gamma) v \frac{d v}{d x}+(1-\gamma) \tau \frac{d v}{d x}+2 v \xi\left(\frac{d v}{d x}-\frac{d \xi}{d x}\right)\right] \\
&(3 \gamma+5) \frac{d \xi}{d x}-(\gamma+5) \frac{d v}{d x}+5(1-\gamma) \frac{d \tau}{d x}=\frac{\varepsilon u_{0}}{A n_{0}}\left[4 \gamma(\gamma+4) \frac{d^{2} \xi}{d x^{2}}+\left(\gamma^{2}+8 \gamma+5\right) \frac{d^{2} v}{d x^{2}}+\right. \\
&\left.+\left(10-2 \gamma-8 \gamma^{2}\right) \frac{d^{2} \tau}{d x^{2}}\right]+\varepsilon\left[(\gamma+5) v \frac{d v}{d x}-5(1-\gamma) \xi \frac{d \tau}{d x}+\right. \\
&\left.+5(1-\gamma) \tau\left(\frac{d v}{d x}-\frac{d \xi}{d x}\right)+2 v \xi\left(\frac{d v}{d x}-\frac{d \xi}{d x}\right)\right]
\end{aligned}
$$


Putting the series $(5,25)$ in the equations $(5,31)$ and $(5,32)$ we have upto $O(\varepsilon)$

$$
\begin{aligned}
& 2 \gamma \frac{d \xi_{1}}{d x}-(1+\gamma) \frac{d v_{1}}{d x}+(1-\gamma) \frac{d \tau_{1}}{d x}=\frac{u_{0}}{A n_{0}}\left(\gamma-\frac{1}{3}\right) \frac{d^{2} \xi_{0}}{d x^{2}}+\frac{d \xi_{0}^{2}}{d x} \\
& (3 \gamma+5) \frac{d \xi_{1}}{d x}-(\gamma+5) \frac{d v_{1}}{d x}+5(1-\gamma) \frac{d \tau_{1}}{d x}=\frac{u_{0}}{A n_{0}}(\gamma-1) \frac{d^{2} \xi_{0}}{d x^{2}}+5 \frac{d \xi_{0}^{2}}{d x}
\end{aligned}
$$

with

$$
(5-3 \gamma) \frac{d \xi_{0}}{d x}=0
$$

From equation $(5,35)$ we have

$$
\gamma=\frac{5}{3} \text { for } \frac{d \xi_{0}}{d x} \neq 0
$$

The value which comes out of the analysis-confirming the physical fact underlying the BGK model itself. Moreover, in order to satisfy the Rankine-Hugoniot relations we must have $v_{0}=\tau_{0}=\xi_{0}$ and this fact has been exploited in the above equations $(5,33)$ an $(5,34)$. Taking into account the remark made at the end of the section $(5.2)$, i.e. one of the integral equations can be eliminated with the aid of conservation of mass relation, we finally arrive at the following governing equations:

$$
\begin{aligned}
& \frac{10}{3} \frac{d \xi_{1}}{d x}-\frac{8}{3} \frac{d v_{1}}{d x}-\frac{2}{3} \frac{d \tau_{1}}{d x}=\frac{u_{0}}{A n_{0}} \cdot \frac{4}{3} \frac{d^{2} \xi_{0}}{d x^{2}}+\frac{d \xi_{0}^{2}}{d x} \\
& 10 \frac{d \xi_{1}}{d x}-\frac{20}{3} \frac{d v_{1}}{d x}-\frac{10}{3} \frac{d \tau_{1}}{d x}=\frac{u_{0}}{A n_{0}} \cdot \frac{2}{3} \frac{d^{2} \xi_{0}}{d x^{2}}+5 \frac{d \xi_{0}^{2}}{d x} \\
& \xi_{1}-v_{1}=\xi_{0}^{2}
\end{aligned}
$$

for the value of $\gamma$ given by $(5,36)$.

With the help of $(5,39)$ equations $(5,37)$ and $(5,38)$ reduce to

$$
\begin{aligned}
& \frac{10}{3} \frac{d \xi_{0}^{2}}{d x}+\frac{2}{3}\left(\frac{d v_{1}}{d x}-\frac{d \tau_{1}}{d x}\right)=\frac{u_{0}}{A n_{0}} \cdot \frac{4}{3} \frac{d^{2} \xi_{0}}{d x^{2}}+\frac{d \xi_{0}^{2}}{d x} \\
& 10 \frac{d \xi_{0}^{2}}{d x}+\frac{10}{3}\left(\frac{d v_{1}}{d x}-\frac{d \tau_{1}}{d x}\right)=\frac{u_{0}}{A n_{0}} \cdot \frac{2}{3} \frac{d^{2} \xi_{0}}{d x^{2}}+5 \frac{d \xi_{0}^{2}}{d x}
\end{aligned}
$$


From equations $(5,40)$ and $(5,41)$ it follows that

$$
\frac{20}{3} \frac{d \xi_{0}^{2}}{d x}=\frac{u_{0}}{A n_{0}} \cdot 6 \frac{d^{2} \xi_{0}}{d x^{2}}
$$

or

$$
\frac{d^{2} \xi_{0}}{d x^{2}}=\frac{10}{9} \frac{A n_{0}}{u_{0}} \frac{d \xi_{0}^{2}}{d x}
$$

After integration equation $(5,43)$ results as

$$
\xi_{0}=a_{1} \cdot \frac{1-c \exp \left\{\frac{20 A n_{0} a_{1} x}{9 u_{0}}\right\}}{1+c \exp \left\{\frac{20 A n_{0} a_{1} x}{9 u_{0}}\right\}},
$$

where $a_{1}$ and $c$ are arbitrary constants. This completes the solution of the problem. The solution $(5,44)$ can be put in more elegant form by choosing the origin $x=0$ as the point where $\xi_{0}=(1-c) /(1+c)$, we obtain $a_{1}=1$. By virtue of which $(5,44)$ becomes

$$
\xi_{0}=\frac{1-c \exp \left\{\frac{20 A n_{0} x}{9 u_{0}}\right\}}{1+c \exp \left\{\frac{20 A n_{0} x}{9 u_{0}}\right\}},
$$

where $c$ is an arbitrary constant. 


\section{APPENDIX A}

\section{A. 1. Expansion of $n, T$ and $u$ in terms of the parameter $\varepsilon$.}

We write $n$ as

$$
\begin{aligned}
n & =\frac{n_{1}+n_{2}}{2}+\frac{n_{1}-n_{2}}{2} v \\
& =\frac{n_{1}+n_{2}}{2}\left(1+\frac{n_{1}-n_{2}}{n_{1}+n_{2}} v\right) \\
& =n_{0}\left(1+\frac{u_{2}-u_{1}}{u_{1}+u_{2}}\right) \text { for } n_{1} u_{1}=n_{2} u_{2}
\end{aligned}
$$

Hence

$$
n=n_{0}(1-\varepsilon v)
$$

Similarly

$$
\begin{aligned}
T & =\frac{T_{1}+T_{2}}{2}+\frac{T_{1}-T_{2}}{2} \tau \\
& =T_{0}\left(1+\frac{T_{1}-T_{2}}{T_{1}+T_{2}} \tau\right)
\end{aligned}
$$

We have to express $\left(T_{1}-T_{2}\right) /\left(T_{1}+T_{2}\right)$ in powers of $\varepsilon$ and in order to do that we use the normal shock relations. We know that

$$
\frac{u_{1}-u_{2}}{u_{1}+u_{2}}=\varepsilon
$$

and which can be simplified to give the relation

$$
\frac{u_{2}}{u_{1}}=\frac{1-\varepsilon}{1+\varepsilon}
$$

Further the normal shock relations are

$$
\frac{u_{2}}{u_{1}}=\frac{\gamma-1}{\gamma+1}+\frac{2}{(\gamma+1) M_{1}^{2}}
$$




$$
\frac{p_{2}}{p_{1}}=\frac{2 \gamma}{\gamma+1} M_{1}^{2}-\frac{\gamma-1}{\gamma+1}
$$

and

$$
\frac{T_{2}}{T_{1}}=\frac{p_{2}}{p_{1}} \cdot \frac{u_{2}}{u_{1}}
$$

From (A3) and (A4) we obtain

$$
M_{1}^{2}=\frac{1+\varepsilon}{1-\varepsilon \gamma}
$$

The substitution of (A7) in (A5) yields

$$
\frac{p_{2}}{p_{1}}=\frac{1+\gamma \varepsilon}{1-\varepsilon \gamma}
$$

on account of (A3) and (A8), the relation (A6) becomes

$$
\frac{T_{2}}{T_{1}}=\frac{(1+\varepsilon \gamma)(1-\varepsilon)}{(1-\varepsilon \gamma)(1+\varepsilon)}
$$

or

$$
\begin{aligned}
\frac{T_{1}-T_{2}}{T_{1}+T_{2}} & =\frac{(1-\varepsilon \gamma)(1+\varepsilon)-(1+\varepsilon \gamma)(1-\varepsilon)}{(1-\varepsilon \gamma)(1+\varepsilon)+(1+\varepsilon \gamma)(1-\varepsilon)} \\
& =\frac{\varepsilon(1-\gamma)}{1-\varepsilon^{2} \gamma} \\
& \cong \varepsilon(1-\gamma) \quad \text { neglecting higher powers of } \varepsilon
\end{aligned}
$$

Thus substituting the value of $\left(T_{1}-T_{2}\right) /\left(T_{1}+T_{2}\right)$ from (A9) in (A2) we obtain

$$
T=T_{0}[1+\varepsilon(1-\gamma) \tau]
$$

Carrying out the similar procedure we have

$$
u=u_{0}(1+\varepsilon \xi)
$$




\section{APPENDIX B}

B. 1. Integrals used in the simplification of equations $(5,29)$ and $(5,30)$.

It seems sufficient to mention the type of integrals which we encounter in our analysis.

Firstly, we come across the following type of integral viz.,

$$
\int_{-\infty}^{\bar{x}} L\left(\bar{x}^{\prime}, \varepsilon\right) \exp \left\{-\frac{A n_{0}}{\varepsilon v_{x}}\left(\bar{x}-\bar{x}^{\prime}\right)\right\} d \bar{x}^{\prime}
$$

where $L$ is some function of $\bar{x}^{\prime}$ and $\varepsilon$. Integrating (B1) by parts we get

$$
L(\bar{x}, \varepsilon) \frac{\varepsilon v_{x}}{A n_{0}}-\frac{\varepsilon v_{x}}{A n_{0}} \int_{-\infty}^{\bar{x}} \exp \left\{-\frac{A n_{0}}{\varepsilon v_{x}}\left(\bar{x}-\bar{x}^{\prime}\right)\right\} \frac{d L\left(x^{\prime}, \varepsilon\right)}{d \bar{x}^{\prime}} d \bar{x}^{\prime},
$$

continuing this process we obtain

$$
L(\bar{x}, \varepsilon) \frac{\varepsilon v_{x}}{A n_{0}}-\left(\frac{\varepsilon v_{x}}{A n_{0}}\right)^{2} \frac{d L}{d \bar{x}}+\left(\frac{\varepsilon v_{x}}{A n_{0}}\right)^{2} \int_{-\infty}^{\bar{x}} \exp \left\{-\frac{A n_{0}}{\varepsilon v_{x}}\left(\bar{x}-\bar{x}^{\prime}\right)\right\} \frac{d^{2} L}{d \bar{x}^{\prime 2}} d \bar{x}^{\prime}
$$

It is to be noted, however, that partial integration is repeated so often that the remaining integral is of sufficient small order in $\varepsilon$ so as to be neglegible.

Secondly, we have the integral

$$
\int_{0}^{\infty} x^{n} e^{-\lambda x^{2}} d x
$$

where $n$ is an integer. The results of integration are expressed by the formulae

$$
\left.\begin{array}{l}
\int_{0}^{\infty} x^{2 k} e^{-\lambda x^{2}} d x=\frac{1.3 \ldots(2 k-1)}{2^{k+1}} \sqrt{\frac{\pi}{\lambda^{2 k+1}}} \\
\int_{0}^{\infty} x^{2 k+1} e^{-\lambda x^{2}} d x=\frac{k !}{2 \lambda^{k+1}}
\end{array}\right\}
$$

Further the expressions for $L_{1}$ and $L_{2}$ are as follows: 


$$
\begin{aligned}
& L_{1}=\frac{m u_{0}^{2}}{k T_{0}}\left(v_{x}-u_{0}\right) \xi+\frac{m(1-\gamma)}{2 k T_{0}}\left(v_{x}-u_{0}\right)^{2} \tau+\frac{n_{0}}{\varepsilon v_{x}} \int_{\bar{x}^{\prime}}^{\bar{x}} v d x^{\prime \prime}, \\
& L_{2}=\frac{n_{0}^{2}}{\varepsilon^{2} v_{x}^{2}}\left[\int_{\bar{x}^{\prime}}^{\bar{x}} v d x^{\prime \prime}\right]^{2}+\frac{m n_{0} u_{0}}{k T_{0} \varepsilon v_{x}}\left(v_{x}-u_{0}\right) \xi \int_{\bar{x}^{\prime}}^{\bar{x}} v d x^{\prime \prime}+ \\
& +\frac{m n_{0}(1-\gamma)}{2 k T_{0} \varepsilon v_{x}}\left(v_{x}-u_{0}\right)^{2} \tau \int_{\bar{x}^{\prime}}^{\bar{x}} v d x^{\prime \prime}+\frac{m^{2} u_{0}^{2}}{2 k^{2} T_{0}^{2}}\left(v_{x}-u_{0}\right)^{2} \xi^{2}- \\
& -\frac{m u_{0}^{2} \xi^{2}}{2 k T_{0}}+\frac{m u_{0}^{2}(1-\gamma)}{2 k^{2} T_{0}^{2}}\left(v_{x}-u_{0}\right)^{2} \xi \tau-\frac{m u_{0}(1-\gamma)}{k T_{0}}\left(v_{x}-u_{0}\right) \xi \tau+ \\
& +\frac{m^{2}(1-\gamma)^{2}}{8 k^{2} T_{0}^{2}}\left(v_{x}-u_{0}\right)^{4} \tau^{2}-\frac{m(1-\gamma)^{2}}{2 k T_{0}}\left(v_{x}-u_{0}\right)^{2} \tau^{2}
\end{aligned}
$$




\section{REFERENCES}

1. Chapman, S., and T. G. Cowling (1952) The Mathematical Theory of Non-Uniform Gases. Cambridge University Press.

2. Jeans, J. H. (1954) The Dynamical Theory of Gases. Dover Publications, Inc.

3. Present, R. D. (1958) Kinetic Theory of Gases. McGraw-Hill Book Company, Inc.

4. Grad, H. (1958) Principles of Kinetic Theory of Gases. Handbuch der Physik, vol. 12, Springer.

5. Bhatnagar, P. L., E. P. Gross, and M. Krook (1954) Phys. Rev., 94, 511.

6. SARIN, S. L. (1967) J. Eng. Math., 1, 203.

7. Gross, E. P., and M. Krook (1956) Phys. Rev., 102, 593.

8. Gross, E. P., E. A. Jackson and S. Ziering (1957) Ann. of Physics 1, 141.

9. Glansdorff, P. (1961) In "Rarefied Gas Dynamics" (L. Talbot, Ed.), 475, Academic Press. Also see Phys. Fluids, 5, (1962), 371.

10. ZIERING, S., E. EK, and P. Koch (1961) Phys. Fluids, 4, 975.

11. Burnett, D. (1935) Proc. Lond. Math. Soc., 39, 385.

12. Burnett, D. (1936) Proc. Lond. Math. Soc., 40, 382.

13. Grad, H. (1949) Comm. Pure Appl. Math., 2, 331.

14. Мотт-Sмiтh, H. M. (1951) Phys. Rev. 82, 885.

15. Holway, L. H. (1964) Kinetic Theory of Shock Structure using an Ellipsoidal Distribution Function. Technical Memorandum T-554, Raytheon Co. Also In "Rarefied Gas Dynamics" (J. H. de Leeuw, ed.), I, 193, 1965. Academic Press.

16. LeEs, L. (1959) GALCIT Hypersonic Research Project, Memorandum NO. 51.

17. Beck, J. W. (1965) In "Rarefied Gas Dynamics" (J. H. de Leeuw, ed.), vol. I, Academic Press.

18. SHEN, S. F. (1963) In "Rarefied Gas Dynamics" (J. A. Laurmann, ed.), vol. II, Academic Press.

19. TAKAO, K. (1961) In "Rarefied Gas Dynamics" (L. Talbot, ed.), Academic Press.

20. Ikenberry, E., and C. Truesdell (1956) J. Rat. Mech. Analysis, 5, 1.

21. Rosen, P. (1954) J. Appl. Physics, 25, 336.

22. Rosen, P. (1954) J. Chem. Physics, 22, 1045.

23. Broadwell, J. (1964) J. Fluid Mech., 19, 401.

24. Chandrasekhar, S. (1960) Radiative Transfer. Dover Publications, Inc.

25. Hamel, B. B., and M. Wachman (1965) In "Rarefied Gas Dynamics" (J. H. de Leeuw, ed.), vol. I, Academic Press.

26. Wachman, M., and B. B. Hamel (1967) In "Rarefied Gas Dynamics" (C. L. Brundin, ed.), vol. I, Academic Press.

27. Huang, A. Ben, and Don P. Giddens (1967) In "Rarefied Gas Dynamics" (C. L. Brundin, ed.), vol. I, Academic Press.

28. NordsiecK, A., and B. L. Hicks (1967) In "Rarefied Gas Dynamics" (C. L. Brundin, ed.), vol. I, Academic Press.

29. KaHn, F. D. (1955) I.A.U. Symposium Series, Symposium Number 2, held at Cambridge, England, 1953. North Holland Publishing Co.

30. Welander, P. (1954) Arkiv Fysik, 7, 507.

31. Maxwell, J. C. (1867) Phil. Trans. Roy. Soc. Lond., 157, 49.

32. Aвramowitz, M. (1953) J. Math and Physics, 32, 188.

33. Huang, A. B. (1967) J. Math and Physics, 46, 107.

34. Hayes, W. D. (1960) Gas Dynamic Discontinuities. Princeton University Press.

35. Lighthill, M. J. (1956) Surveys in Mechanics (G. K. BAtchelor and R. M. Davies, ed.), Cambridge University Press.

36. Sherman, F. S., and L. Talbot (1960) In "Rarefied Gas Dynamics" (F. M. Devienne, ed.), Pergamon Press. 
37. Chahine, M. T. (1963) In "Rarefied Gas Dynamics" (J. A. Laurmann, ed.), vol. I, Academic Press.

38. Haviland, J. K. (1963) In "Rarefied Gas Dynamics" (J. A. Laurmann, ed.), vol. I, Academic Press.

39. Chahine, M. T., and R. Narasimha (1965) In "Rarefied Gas Dynamics" (J. H. de Leeuw, ed.), vol. I, Academic Press.

40. OGuchi, P., (1965) In "Rarefied Gas Dynamics" (J. H. de Leeuw, ed.), vol. I, Academic Press.

41. Holway, H. L. (1965) In "Rarefied Gas Dynamics" (J. H. de Leeuw, ed.), vol. I, Academic Press.

42. Liepmann, H. W., R. Narasimha, and M. T. Chahine (1962) Phys. Fluids, 5, 1313.

43. TAKano, A. (1962) Trans. Jap. Soc. Aero. and Sp. Sc., 5, 1. 


\section{SUMMARY}

The object of this thesis is the development of new techniques for solving the Boltzmann equation and their applications to certain flow problems. A monatomic and single-component gas is considered.

Preliminary remarks are given in chapter 1 . They deal in a cursory manner with the historical aspects and the aims of the kinetic theory of gases. Plan of the thesis is also outlined in this chapter.

Chapter 2 deals with the general introduction of the existing techniques for solving the Boltzmann equation. The various techniques for solving the Boltzmann equation has been categorized only under five heads. Comments regarding their applicability are added, wherever necessary.

The collision of gas clouds is considered in chapter 3. The general expressions for the density and the temperature rise are evaluated during the collision process. The results for the collision of two clouds with equal densities are deduced from the general results.

Chapter 4 consists of two parts. Part I deals with the presentation of a new form of the distribution function and its possible applications to problems in kinetic theory of gases. Part II is an illustration of its application to linearized plane couette flow.

Chapter 5 deals with the problem of kinetic theory of shock structure. Only weak shock is considered. A system of three singular, non-linear integral equations is derived for density, temperature and average velocity by using BGK model. The equations are expressed in terms of the small parameter and their simplification leads to differential equations. The calculations are carried upto the first approximation. 



\section{SAMENVATTING}

In dit proefschrift worden nieuwe methoden ontwikkeld om oplossingen van de vergelijking van Boltzmann te vinden, terwijl verder deze methoden worden toegepast op bepaalde stromingsproblemen. Beschouwd wordt een een-atomig gas, bestaande uit één component.

In hoofdstuk 1 worden de geschiedenis en het doel van de kinetische gastheorie kort beschreven. Tevens is in dit hoofdstuk een overzicht van het proefschrift gegeven.

In hoofdstuk 2 worden de bestaande oplossingsmethoden voor de vergelijking van Boltzmann besproken. Een classificatie van deze methoden in vijf groepen wordt gegeven en waar nodig worden de beperkingen in hun toepassingsmogelijkheden aangegeven.

In hoofdstuk 3 wordt het op elkaar stromen van twee gaswolken beschouwd. De temperatuurstijging en de dichtheid gedurende het ontmoetingsproces worden bepaald. In het speciale geval, dat de dichtheden van de twee gassen voor de ontmoeting gelijk zijn, wordt aangetoond, dat de dichtheid van het mengsel na een oneindig lange tijd nadert tot tweemaal de dichtheid voor de ontmoeting.

Hoofdstuk 4 bestaat uit twee delen. Het eerste deel bevat een nieuwe vorm voor de verdelingsfunctie terwijl mogelijke toepassingen hiervan in de kinetische gastheorie worden aangegeven. Als illustratie behandelt het tweede deel de toepassing op de gelineariseerde vlakke Couette-stroming.

In hoofdstuk 5 wordt de gaskinetische structuur van zwakke schokgolven onderzocht. Een systeem van drie singuliere, niet-lineaire integraalvergelijkingen voor de dichtheid, temperatuur en gemiddelde snelheid wordt afgeleid, gebruikmakend van het BGK model. Door een parameter, die klein is voor zwakke schokgolven, in te voeren in deze integraalvergelijkingen en alleen de hoofdtermen te beschouwen worden differentiaalvergelijkingen verkregen, die analytisch worden opgelost. 



\section{STELLINGEN}

I

In certain problems of rarefied gas flows a good approximation is given by assuming that the departure from some equilibrium conditions manifests itself through the perturbation of the velocity distribution functions in only one particular direction.

Abarbanel, S. S. (1967) In Rarefied Gas Dynamics (C. L. Brundin, ed.) Vol. I. Academic Press.

II

The method proposed in chapter 4 of this thesis can be extended to non-linear problems in kinetic theory of gases.

\section{III}

Fluid dynamics' technique used by Bland in studying the time dependent materials can be partially extended to cover the non-linear case.

(i) Bland, D. R. (1965) Inst. Maths. Applics., 1, 56-75.

(ii) Sarin, S. L. (1967) Canad. Cong. Appl. Mech., B 29.

\section{IV}

The method of Lighthill may be employed to evaluate the strain distribution during the formation of a shock wave in case of linear time dependent materials.

Lighthill, M. J. (1956) Surveys in Mechanics, 250-351, Cambridge University Press.

\section{V}

In view of the active interest in rarefied gases it is necessary to develop another possible method of solution of the Boltzmann equation for binary mixtures and to that end the work of Keller may be used as a guideline.

(i) Keller, J. B. (1948) Comm. Appl. Math., 1, 275-285.

(ii) Sarin, S. L., On the Solution of the Boltzmann equation for Binary Mixtures of Rarefied Gases. Accepted for presentation at the Sixth International Symposium on Rarefied Gas Dynamics to be held at M.I.T. Cambridge, Mass. from July 22-26, 1968. To appear in "Rarefied Gas Dynamics" in the series "Advances in Applied Mechanics", Academic Press, New York. 

VI

Mathematics exists simultaneously in two distinct states, both as an art form and as the language of science.

\section{VII}

The official languages (Amendment) Bill adopted last year by both the houses of Indian Parliament is a step in the right direction.

\section{VIII}

Any large-scale drift toward animal slaughter in India before the traction, fuel and manure needs of the production were met would immediately jeopardise the lives of tens of millions of people. 
